\title{
Discovery of STAT3 and Histone Deacetylase (HDAC) Dual-Pathway Inhibitors for the Treatment of Solid Cancer
}

Yuhao Ren ${ }^{1, \mathrm{a}}$, Shanshan $\mathrm{Li}^{1, \mathrm{a}}$, Ren Zhu ${ }^{\mathrm{b}}$, Chengying Wan ${ }^{\mathrm{a}}$, Dongmei Song ${ }^{\mathrm{a}}$, Jiawen Zhu $^{\mathrm{a}}$, Guiping Cai ${ }^{\mathrm{a}}$, Sihui Long ${ }^{\mathrm{b}}$, Wenying $\mathrm{Yu}^{* *, a}$ and Lingyi Kong*,a

aJiangsu Key Laboratory of Bioactive Natural Product Research and State Key Laboratory of Natural Medicines, China Pharmaceutical University, 24 Tong Jia Xiang, Nanjing 210009, People's Republic of China.

${ }^{b}$ Key Laboratory for Green Chemical Process of Ministry of Education, Hubei Key Laboratory of Novel Reactor and Green Chemical Technology, Hubei Engineering Research Center for Advanced Fine Chemicals, School of Chemical Engineering and Pharmacy, Wuhan Institute of Technology, $2061^{\text {st }}$ Rd Optics Valley, East Lake New Technology Development District, Wuhan, Hubei 430205, China.

*Corresponding Author.

**Corresponding Author.

Tel/Fax: +86-25-83271405; E-mails: cpu_lykong@126.com (Lingyi Kong); ywy@cpu.edu.cn (Wenying Yu).

${ }^{1}$ Both authors contributed equally to this paper.

\section{Contents}

- The representative ${ }^{1} \mathrm{H}$ NMR, ${ }^{13} \mathrm{C}$ NMR, IR and HRMS (ESI) spectra of compounds 11-19 and HPLC result of compound 14.

- Figure S1. Pharmacokinetics of $\mathbf{1 4}$ in SD rats. 
The representative ${ }^{1} \mathrm{H}$ NMR, ${ }^{13} \mathrm{C}$ NMR, IR, and HRMS (ESI) spectra of compounds 11-19.

Compound 11

${ }^{1} \mathrm{H}$ NMR of compound 11:

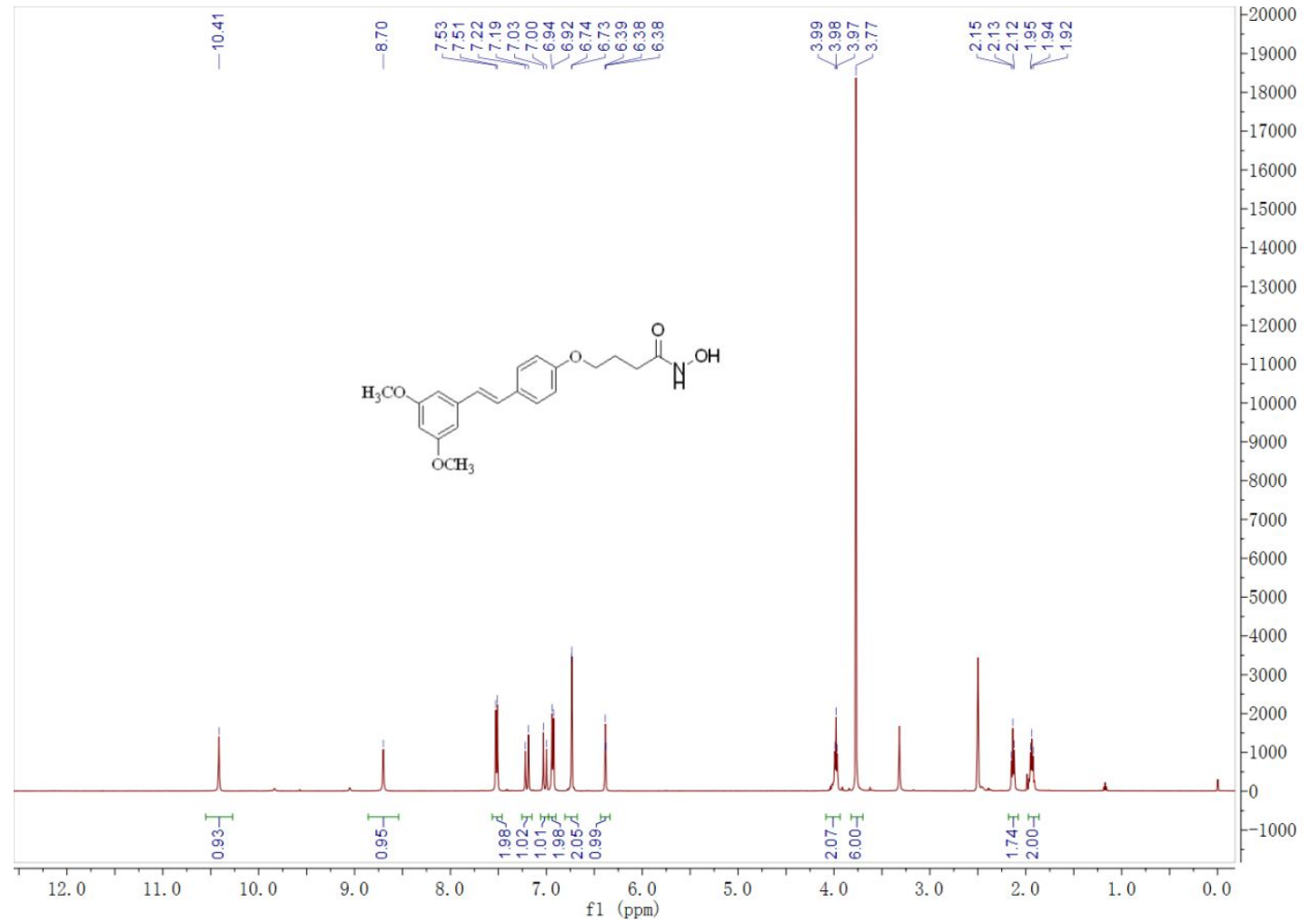


${ }^{13} \mathrm{C}$ NMR of compound $\mathbf{1 1}$ :

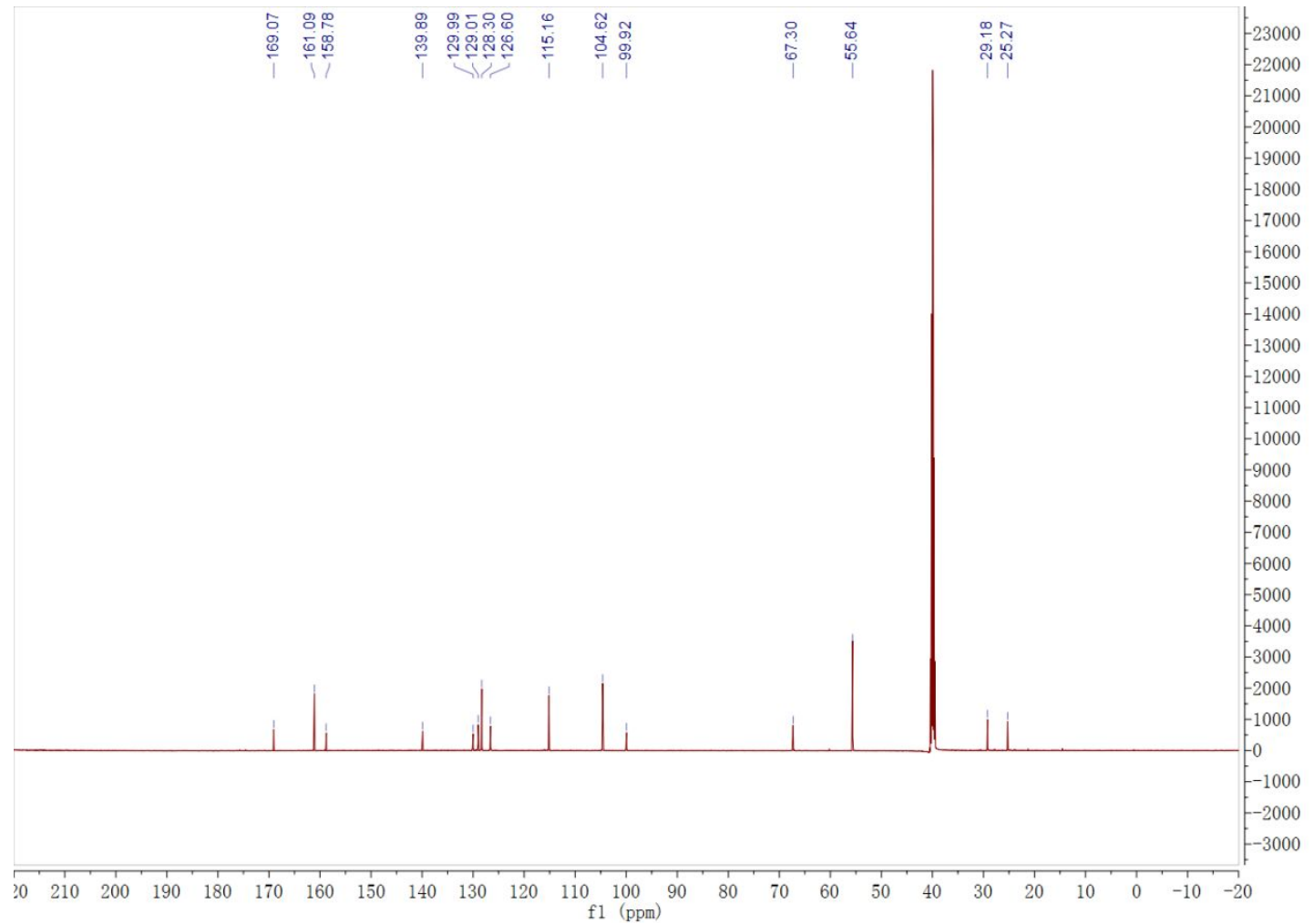

IR of compound 11:

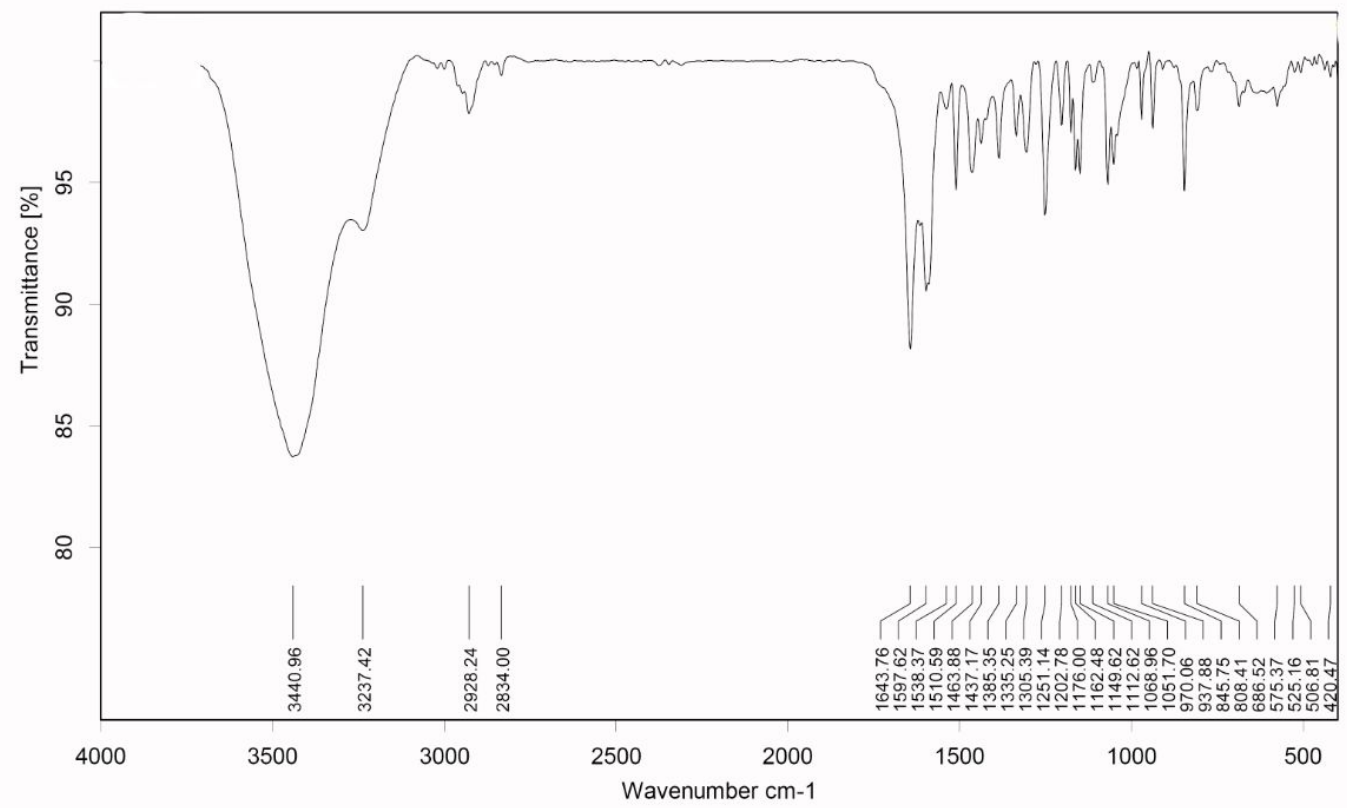

HRMS (ESI) of compound 11: 


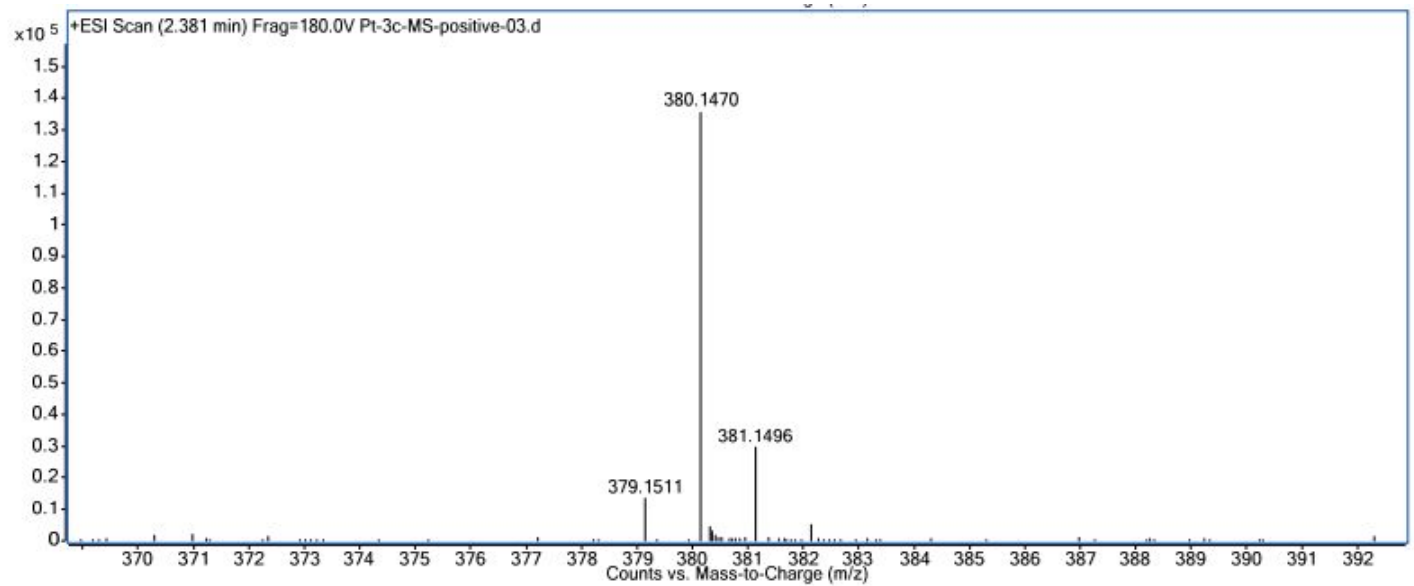

Elemental Composition Calculator

\begin{tabular}{|c|c|c|c|c|c|}
\hline Target m/z: & 380.1470 & Result type: & Positive ions & Species: & {$[\mathrm{M}+\mathrm{Na}]^{+}$} \\
\hline Elements: & \multicolumn{3}{|c|}{$\mathrm{C}(0-80) ; \mathrm{H}(0-120) ; \mathrm{O}(0-30) ; \mathrm{N}(0-5) ; \mathrm{Na}(0-5)$} \\
\hline Ion Formula & \multicolumn{2}{|c|}{ Calculated $\mathbf{~ m / z}$} & PPM Error \\
\hline $\mathrm{C} 20 \mathrm{H} 23 \mathrm{NNaO5}$ & 380.1468 & -0.48 \\
\hline
\end{tabular}




\section{Compound 12}

${ }^{1} \mathrm{H}$ NMR of compound $\mathbf{1 2}$ :

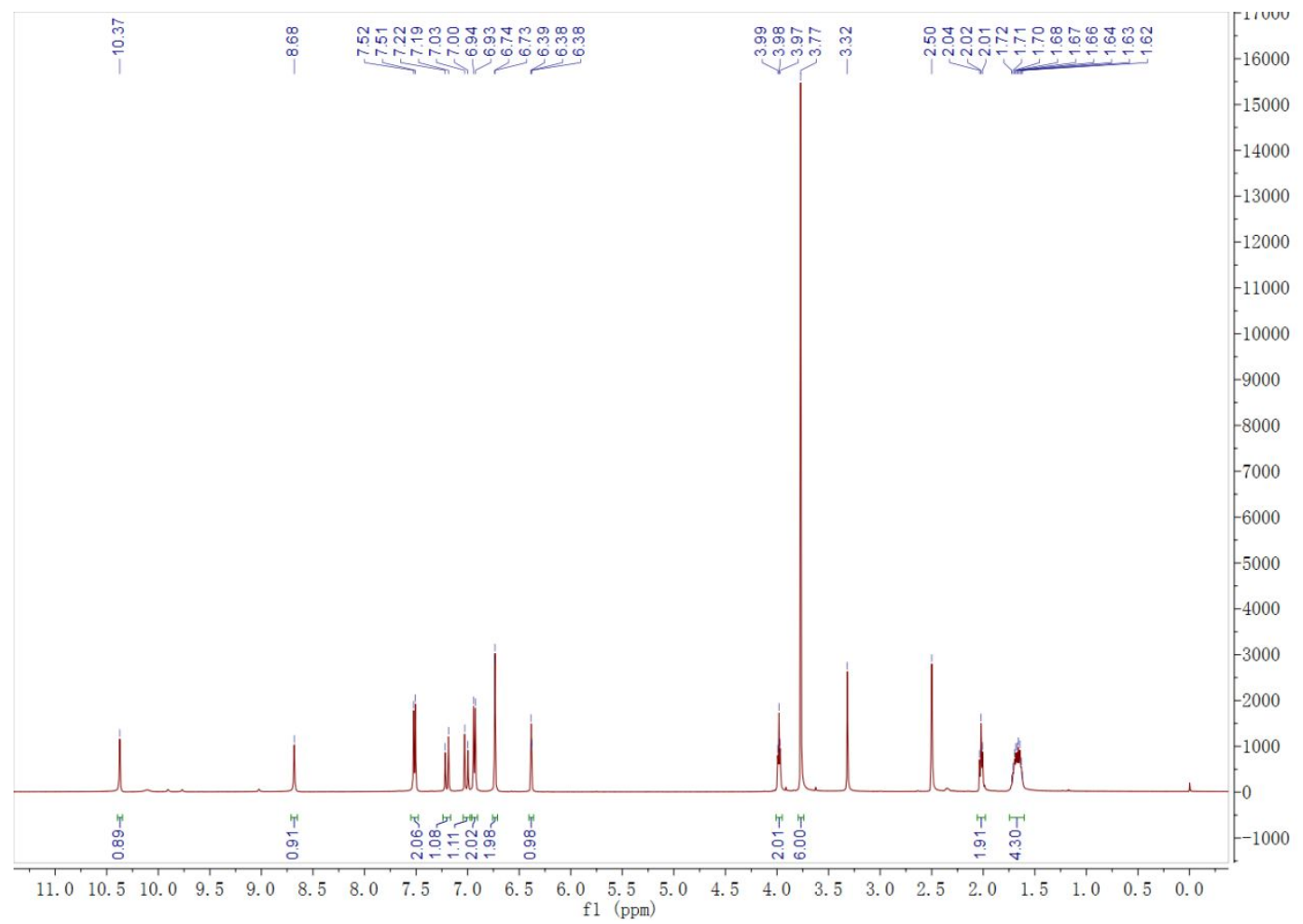


${ }^{13} \mathrm{C}$ NMR of compound $\mathbf{1 2}$ :

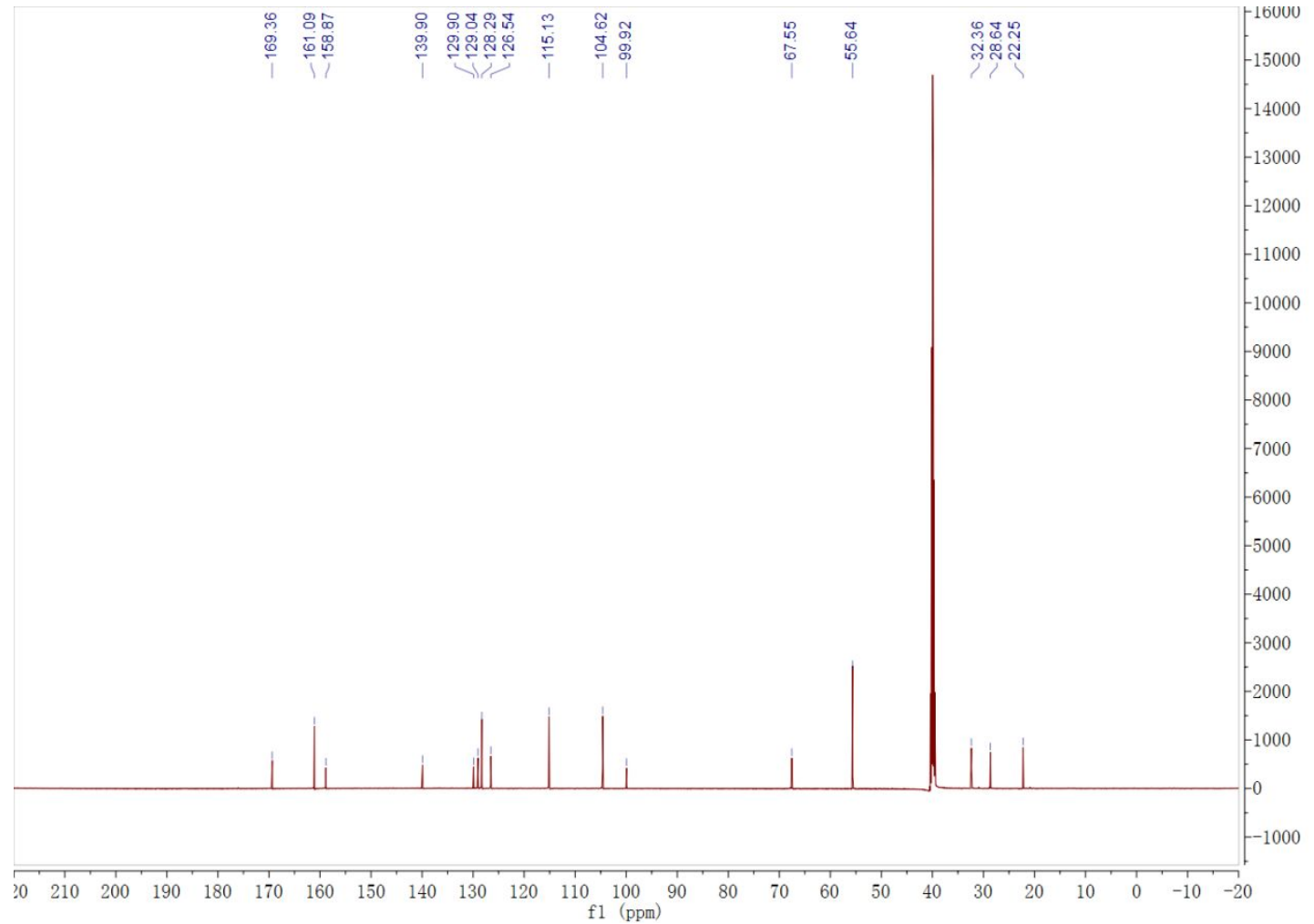

\section{IR of compound 12}

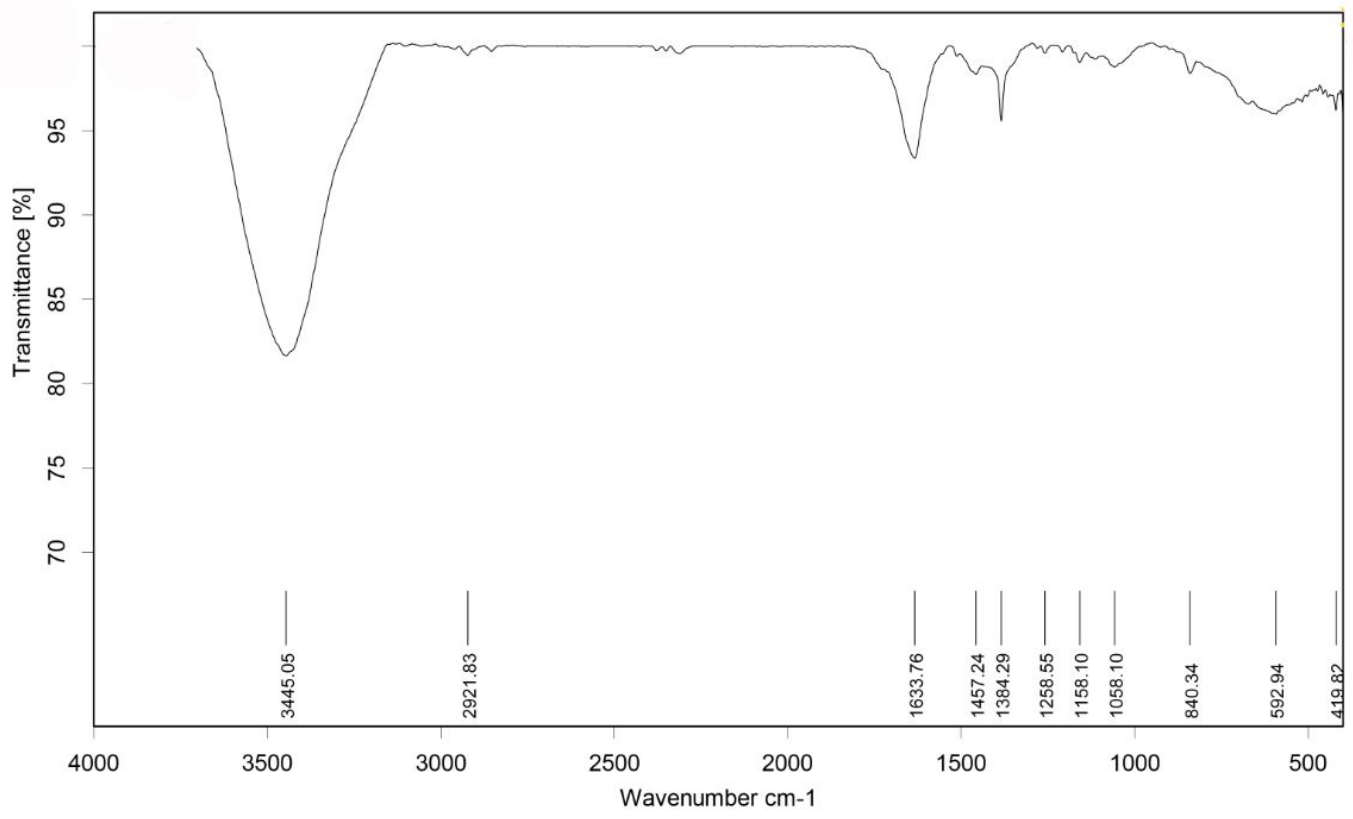


HRMS (ESI) of compound 12:

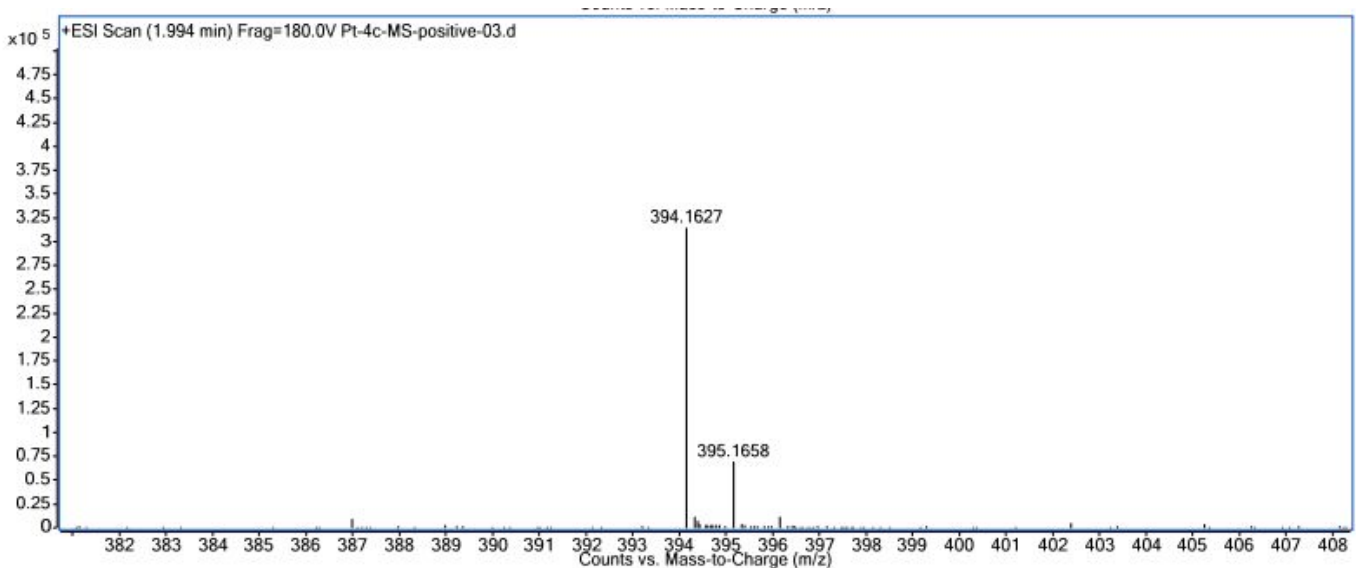

Elemental Composition Calculator

\begin{tabular}{|c|c|c|c|c|c|}
\hline Target $\mathbf{m} / \mathbf{z}$ : & 394.1627 & Result type: & Positive ions & Species: & {$[\mathrm{M}+\mathrm{Na}]^{+}$} \\
\hline \multicolumn{2}{|c|}{ Elements: } & \multicolumn{4}{|c|}{$\mathrm{C}(0-80) ; \mathrm{H}(0-120) ; \mathrm{O}(0-30) ; \mathrm{N}(0-5) ; \mathrm{Na}(0-5)$} \\
\hline \multicolumn{2}{|c|}{ Ion Formula } & \multicolumn{2}{|c|}{ Calculated $\mathbf{m} / \mathbf{z}$} & \multicolumn{2}{|c|}{ PPM Error } \\
\hline \multicolumn{2}{|c|}{$\mathrm{C} 21 \mathrm{H} 25 \mathrm{NNaO} 5$} & \multicolumn{2}{|c|}{394.1625} & \multicolumn{2}{|c|}{-0.51} \\
\hline
\end{tabular}




\section{Compound 13}

${ }^{1} \mathrm{H}$ NMR of compound 13:

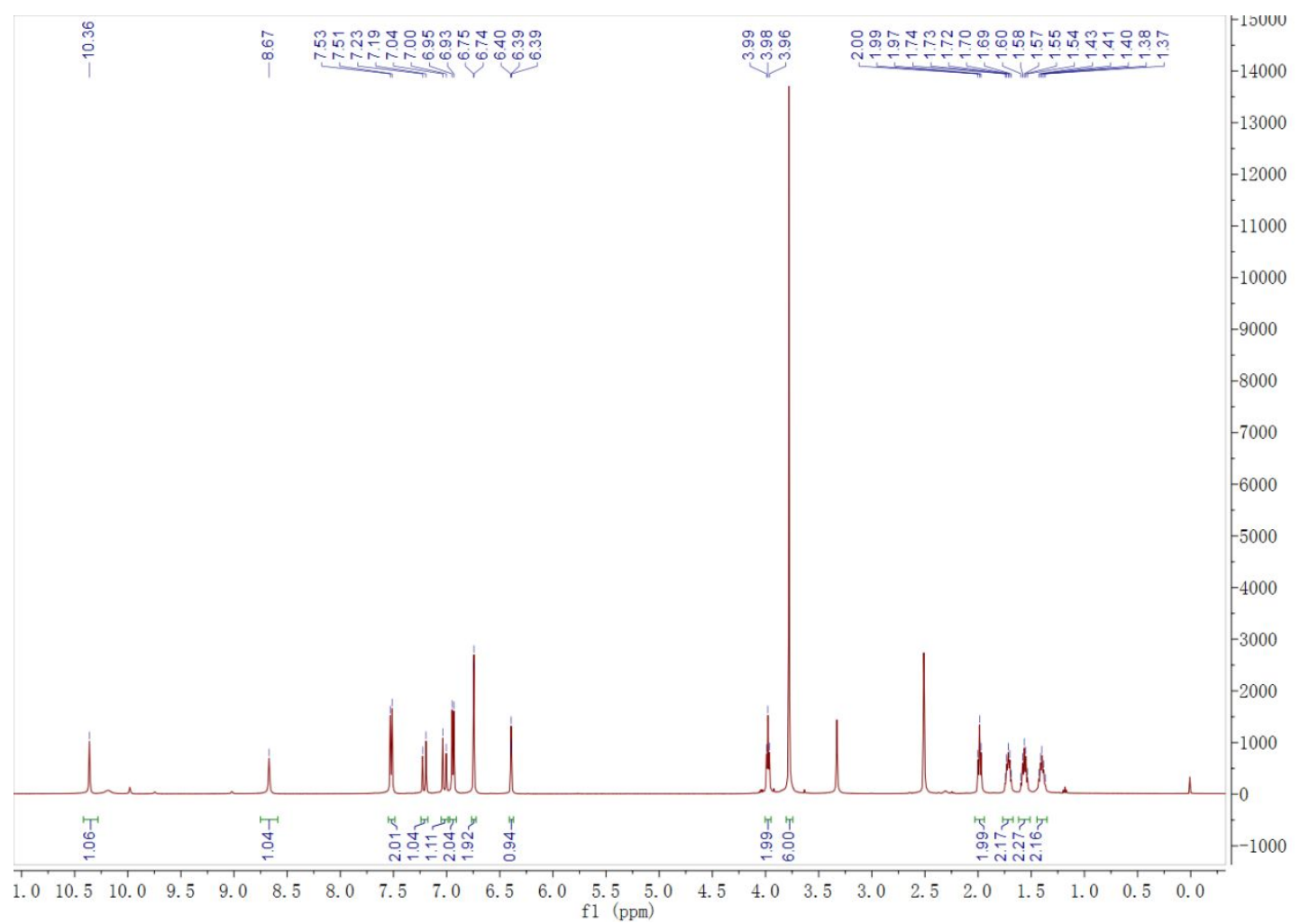


${ }^{13} \mathrm{C}$ NMR of compound $\mathbf{1 3}$ :

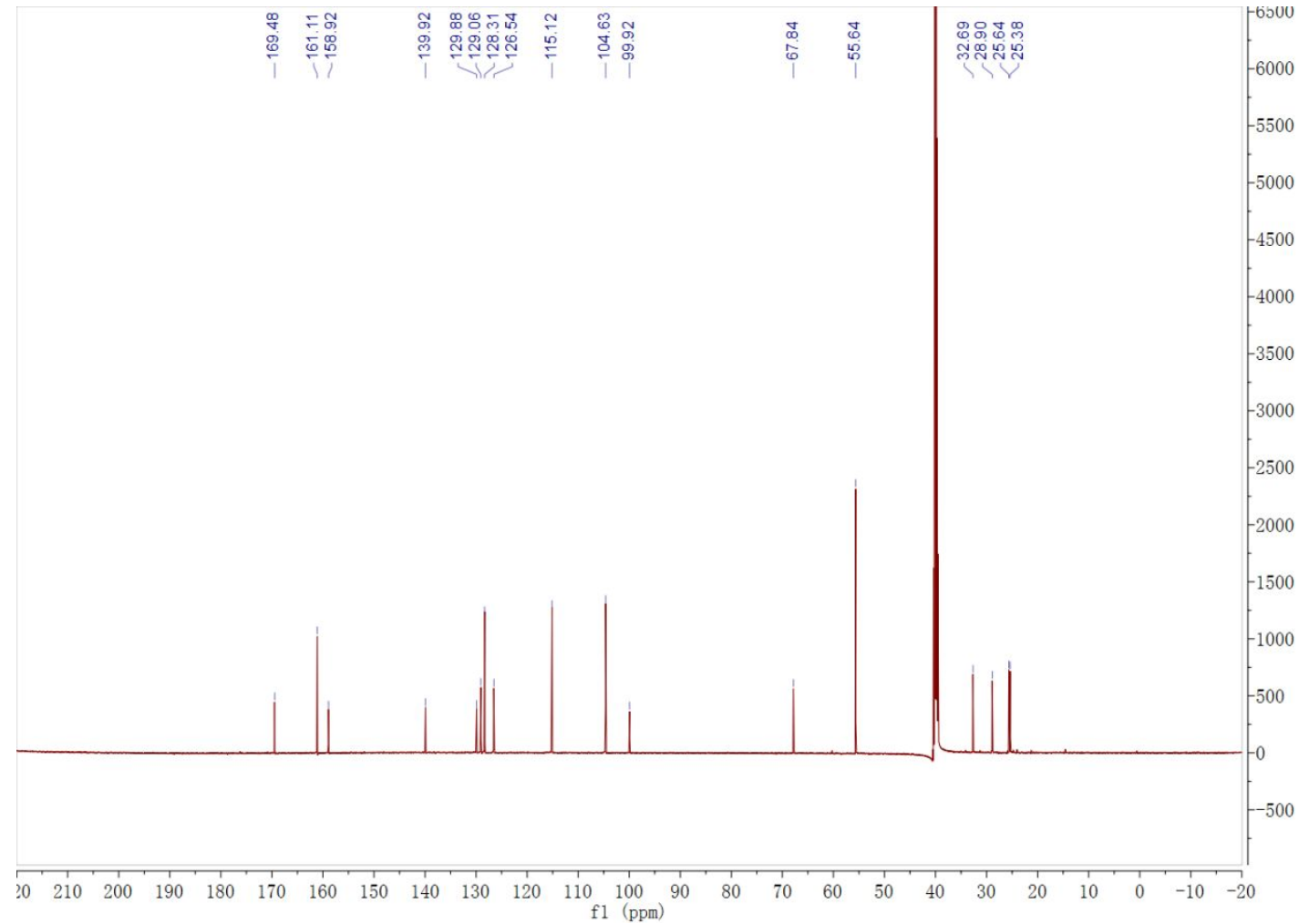

\section{IR of compound 13}

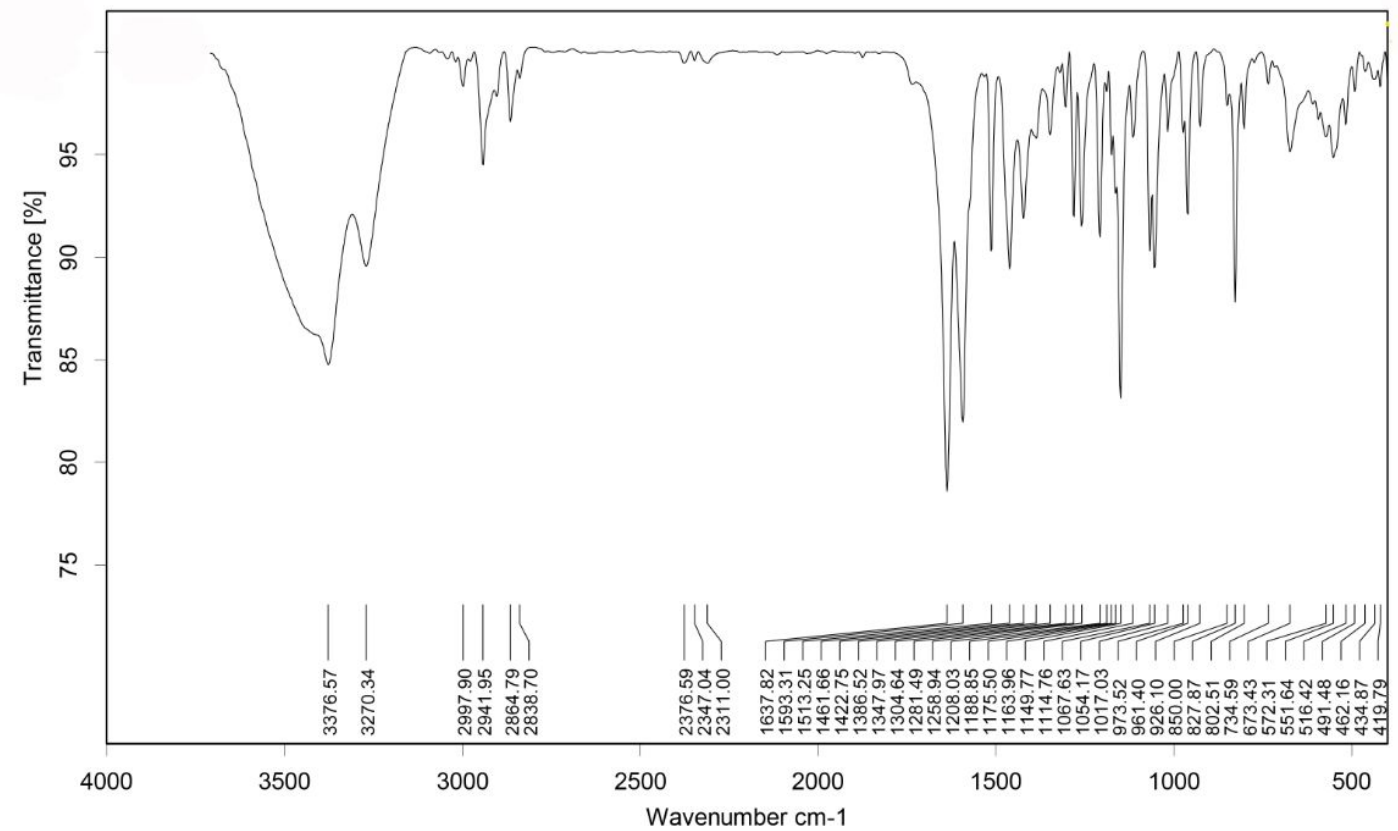


HRMS (ESI) of compound 13:

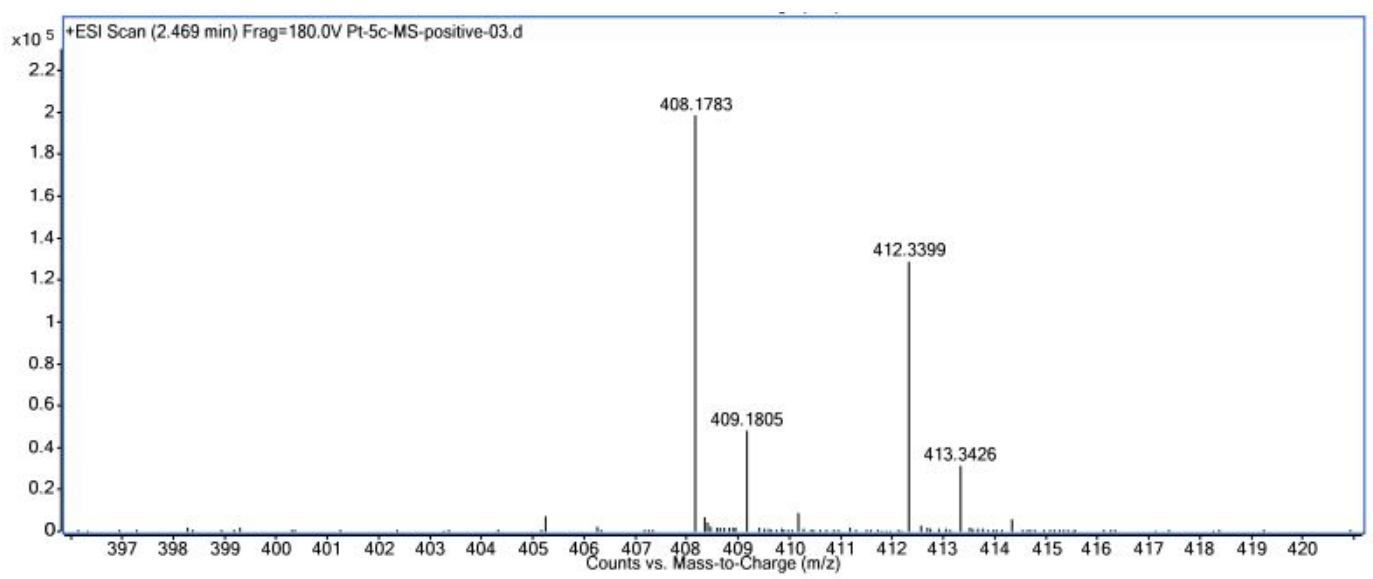

Elemental Composition Calculator

\begin{tabular}{|c|c|c|c|c|c|}
\hline Target $\mathbf{m} / \mathbf{z}$ : & 408.1783 & Result type: & Positive ions & Species: & {$[\mathrm{M}+\mathrm{Na}]^{+}$} \\
\hline \multicolumn{2}{|c|}{ Elements: } & \multicolumn{4}{|c|}{$\mathrm{C}(0-80) ; \mathrm{H}(0-120) ; \mathrm{O}(0-30) ; \mathrm{N}(0-5) ; \mathrm{Na}(0-5)$} \\
\hline \multicolumn{2}{|c|}{ Ion Formula } & \multicolumn{2}{|c|}{ Calculated $\mathbf{m} / \mathbf{z}$} & \multicolumn{2}{|c|}{ PPM Error } \\
\hline \multicolumn{2}{|c|}{$\mathrm{C} 22 \mathrm{H} 27 \mathrm{NNaO} 5$} & \multicolumn{2}{|c|}{408.1781} & \multicolumn{2}{|c|}{-0.44} \\
\hline
\end{tabular}




\section{Compound 14}

${ }^{1} \mathrm{H}$ NMR of compound 14 :

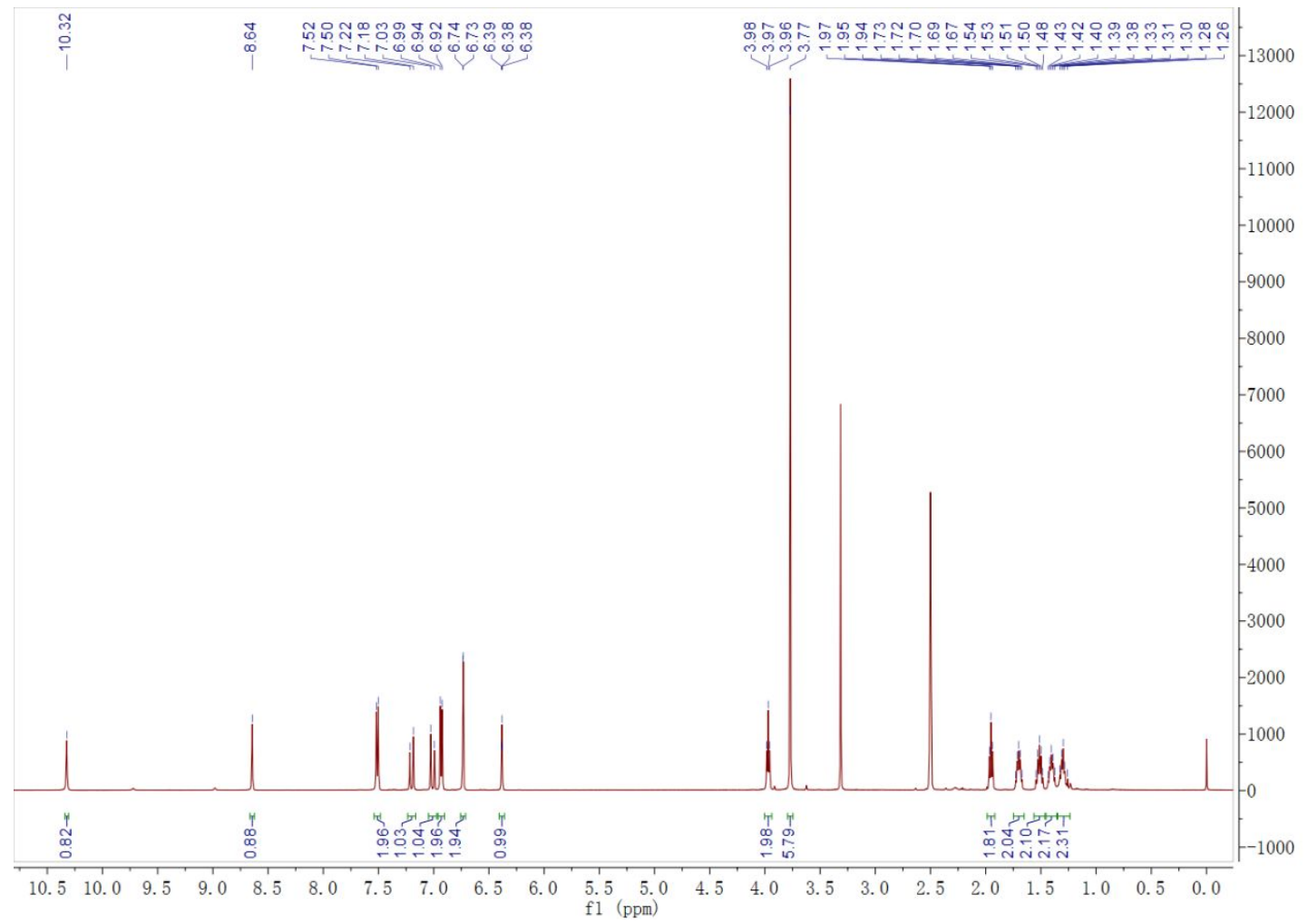


${ }^{13} \mathrm{C}$ NMR of compound 14 :

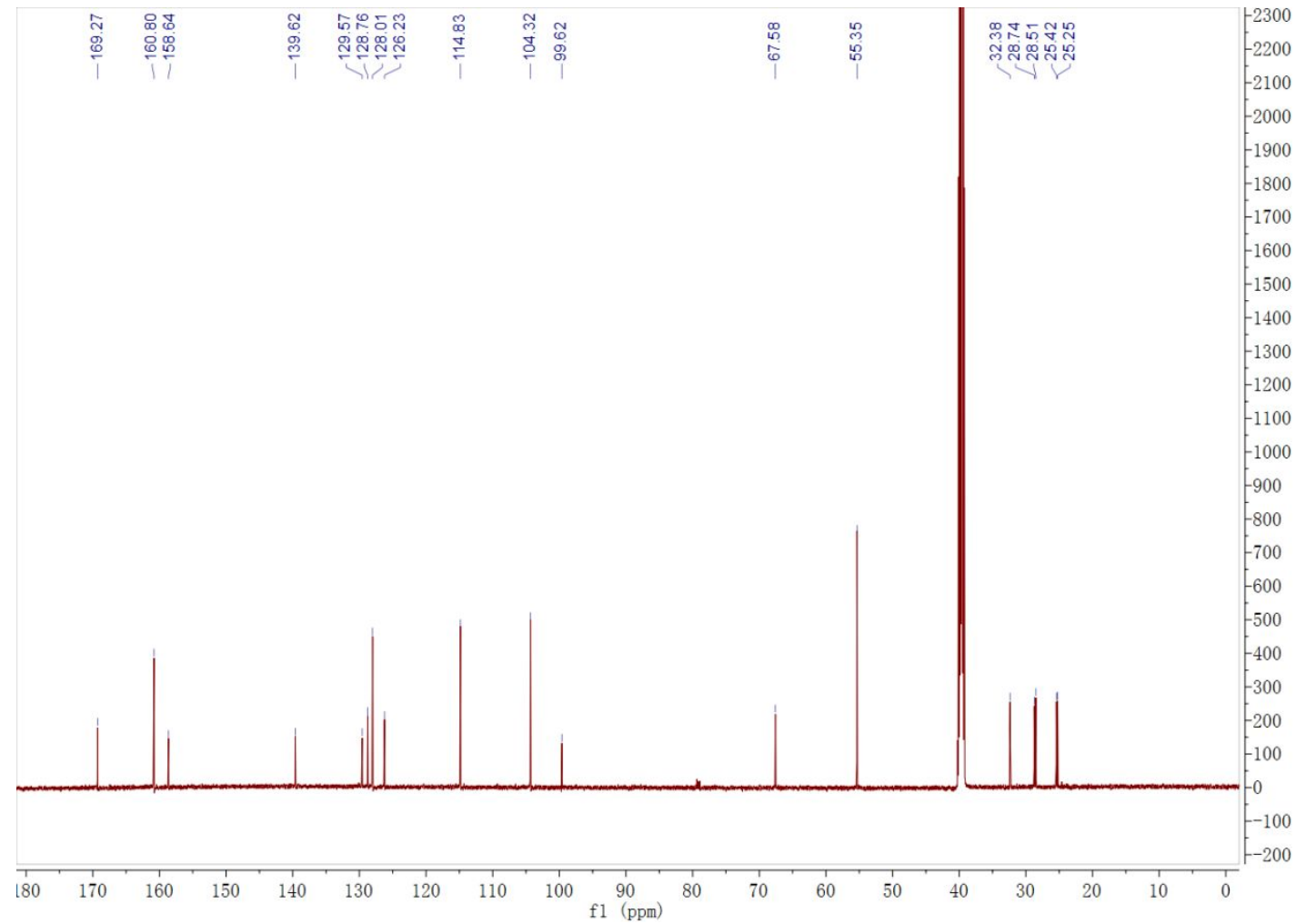

\section{IR of compound 14}

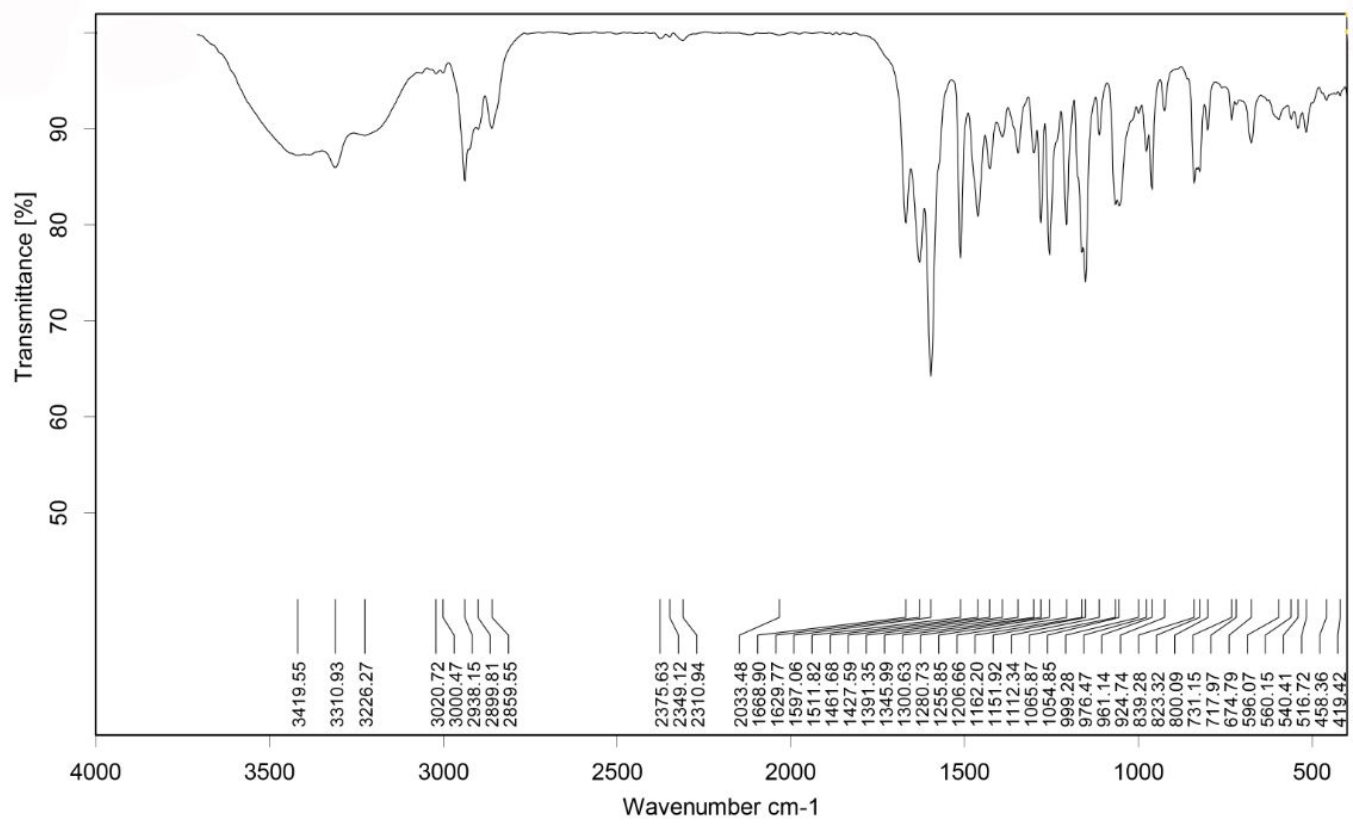


HRMS (ESI) of compound 14

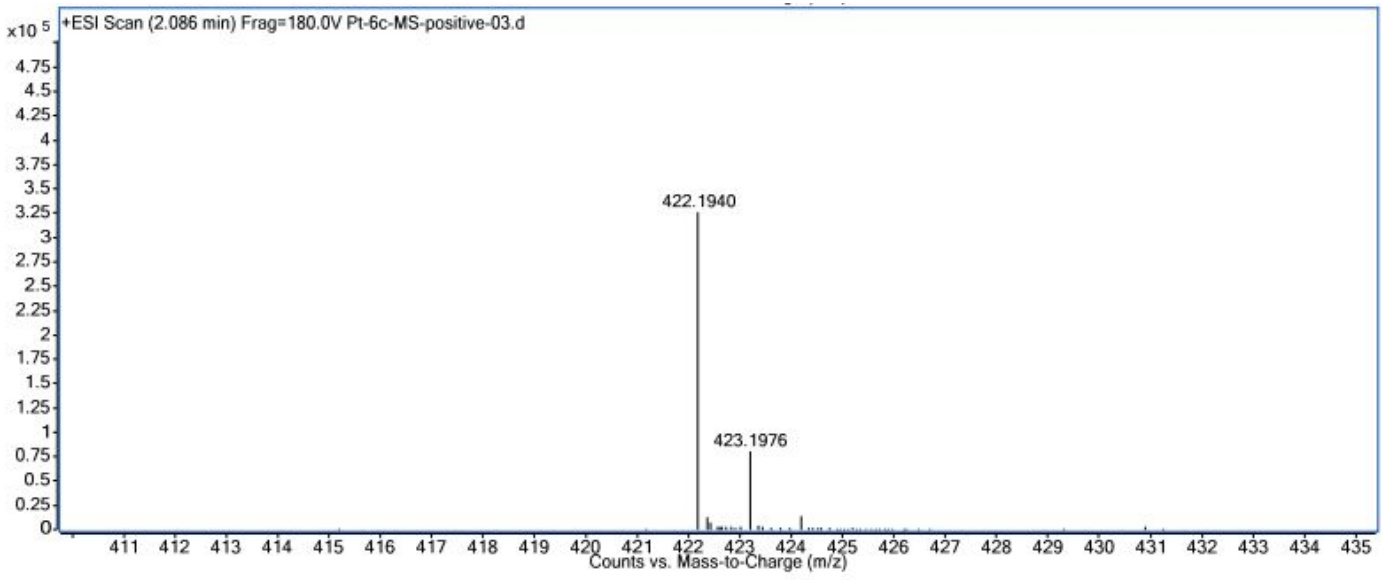

Elemental Composition Calculator

\begin{tabular}{|c|c|c|c|c|c|}
\hline Target $\mathbf{m} / \mathbf{z}$ : & 422.1940 & Result type: & Positive ions & Species: & {$[\mathrm{M}+\mathrm{Na}]^{+}$} \\
\hline \multicolumn{2}{|c|}{ Elements: } & \multicolumn{4}{|c|}{$\mathrm{C}(0-80) ; \mathrm{H}(0-120) ; \mathrm{O}(0-30) ; \mathrm{N}(0-5) ; \mathrm{Na}(0-5)$} \\
\hline \multicolumn{2}{|c|}{ Ion Formula } & \multicolumn{2}{|c|}{ Calculated $\mathbf{m} / \mathbf{z}$} & \multicolumn{2}{|c|}{ PPM Error } \\
\hline \multicolumn{2}{|c|}{$\mathrm{C} 23 \mathrm{H} 29 \mathrm{NNaO} 5$} & \multicolumn{2}{|c|}{422.1938} & \multicolumn{2}{|c|}{-0.54} \\
\hline
\end{tabular}

The HPLC of compound 14:

Purity $=99.25 \%$

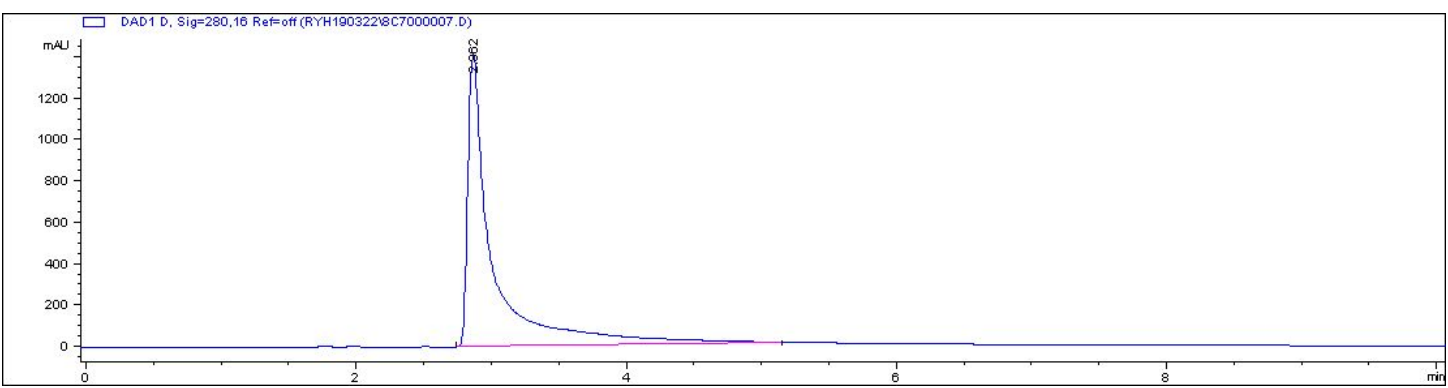




\section{Compound 15}

${ }^{1} \mathrm{H}$ NMR of compound 15

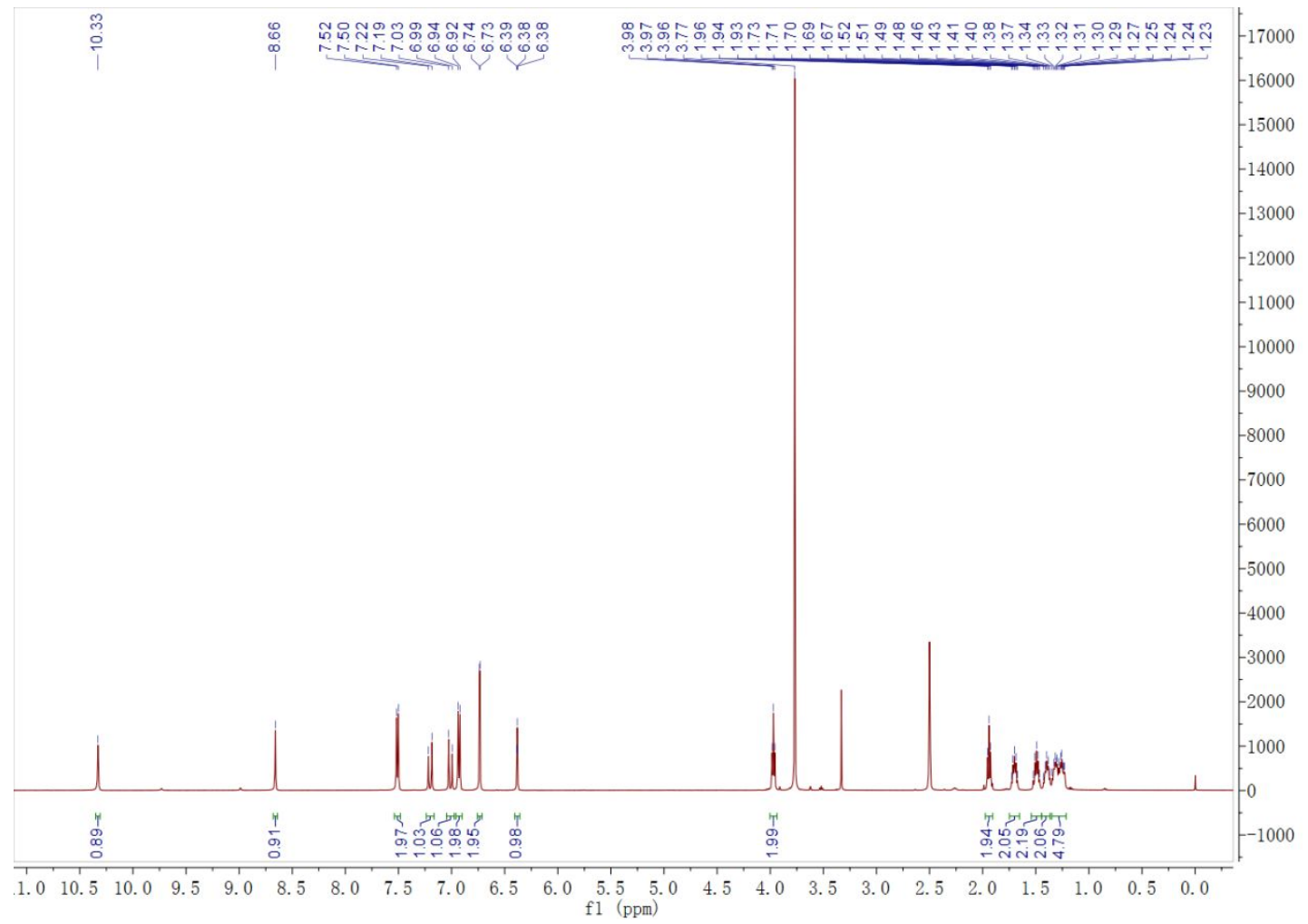


${ }^{13} \mathrm{C}$ NMR of compound $\mathbf{1 5}$ :

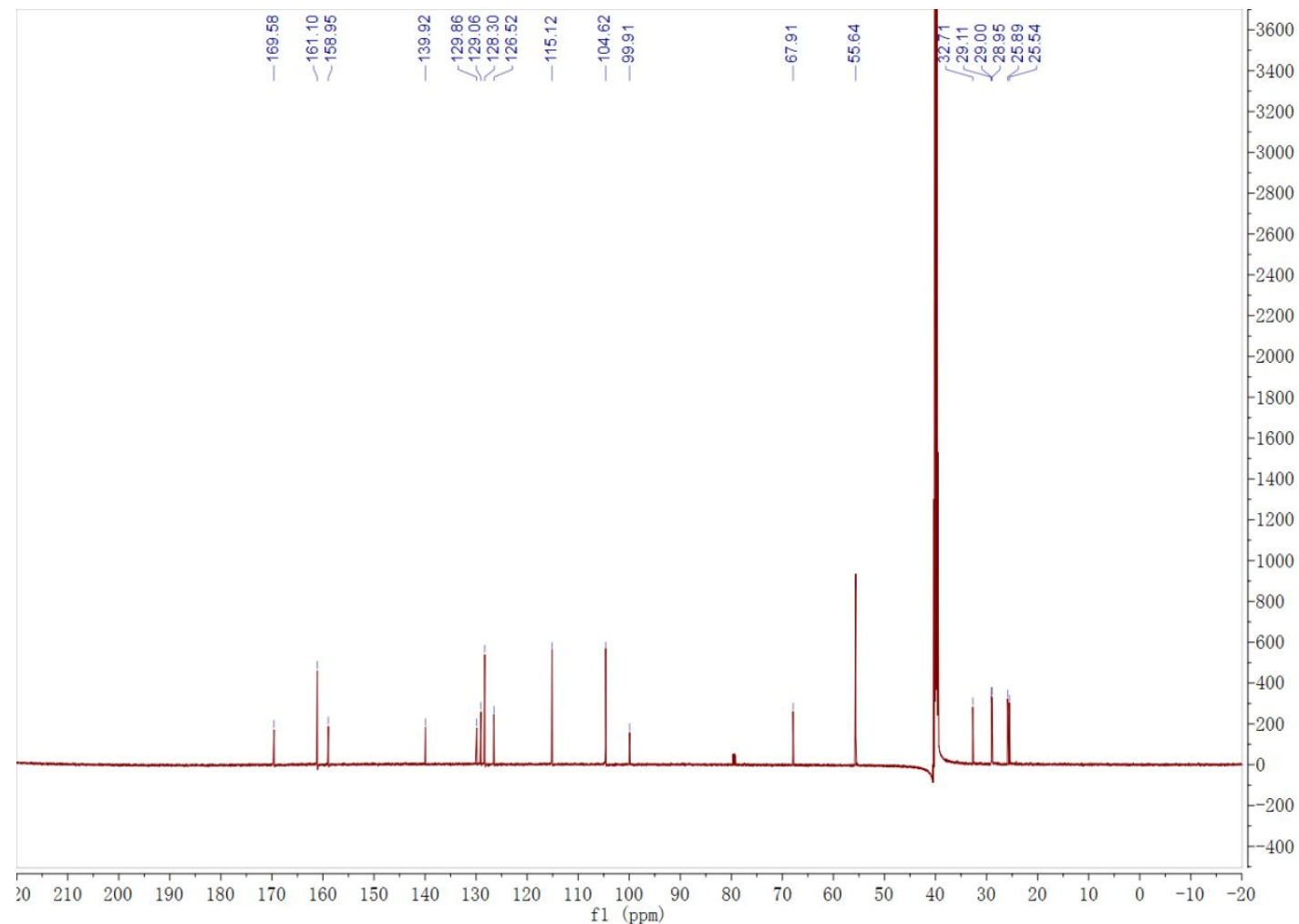

\section{IR of compound 15}

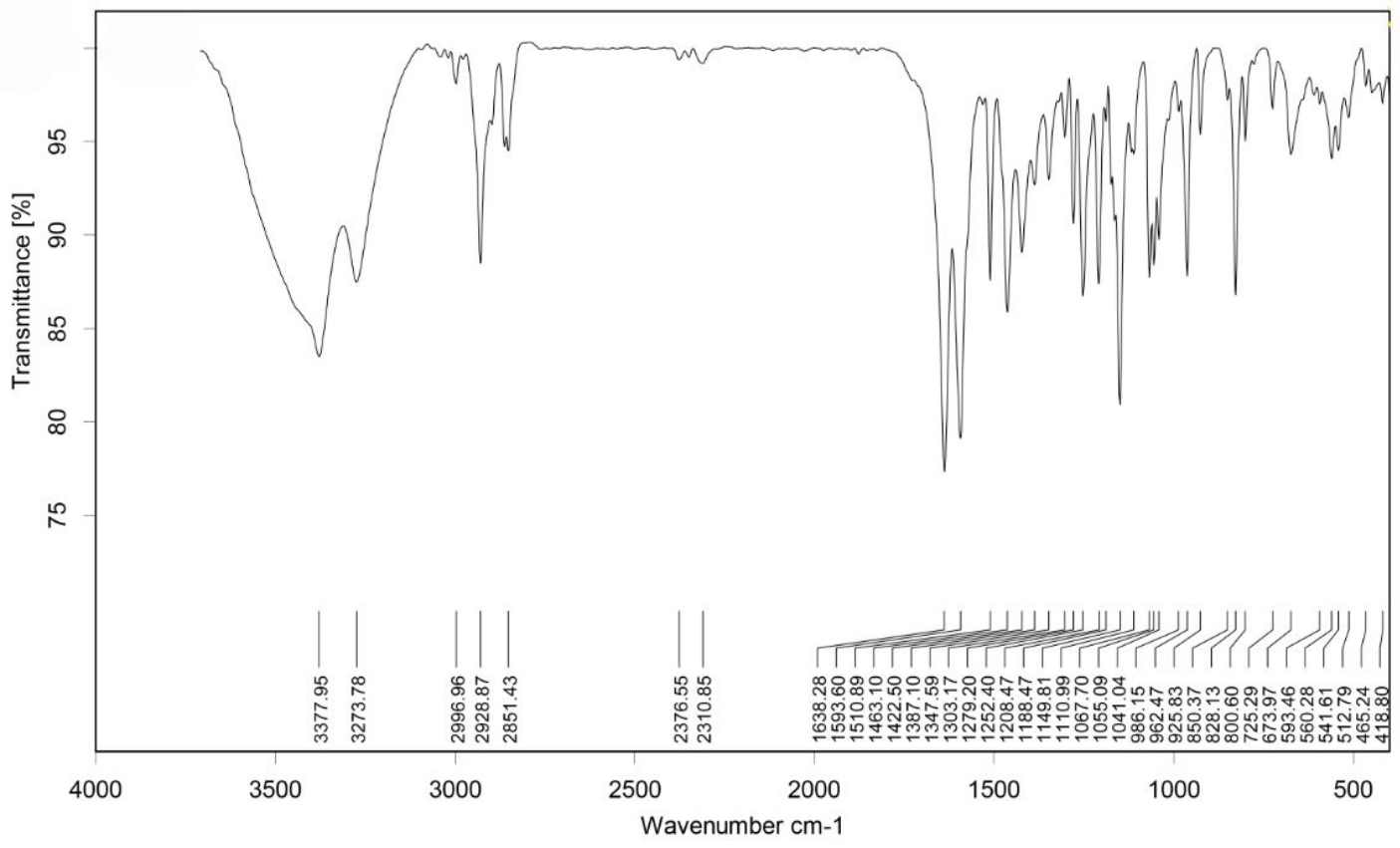


HRMS (ESI) of compound $\mathbf{1 5}$

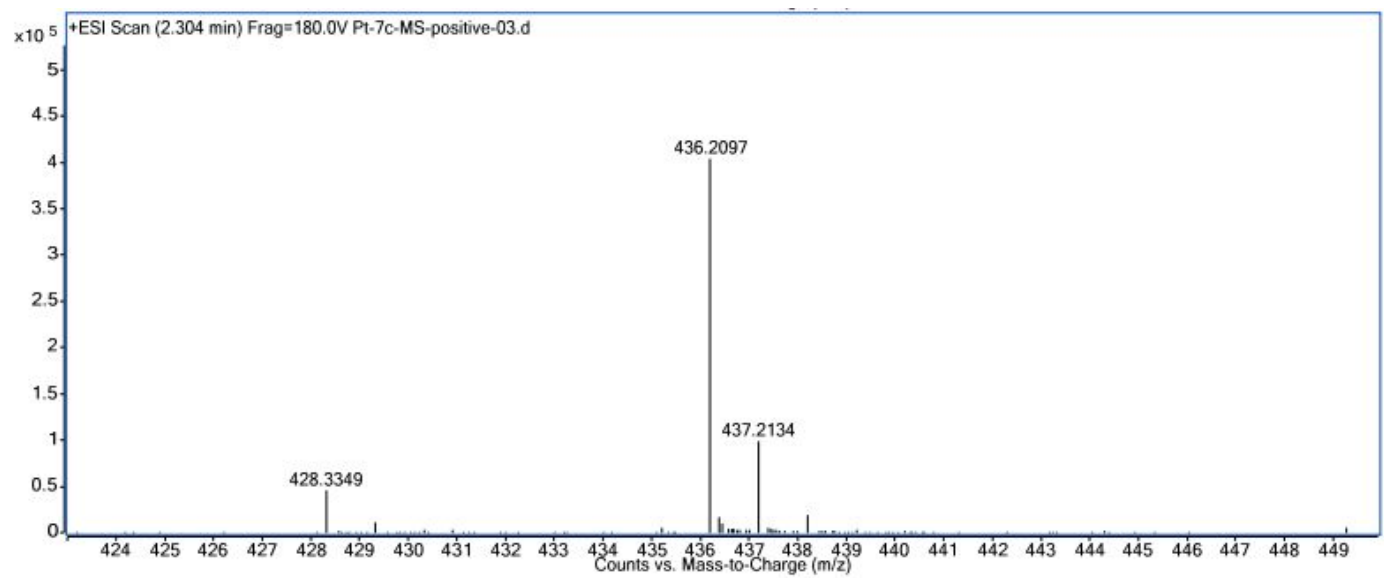

Elemental Composition Calculator

\begin{tabular}{|c|c|c|c|c|c|}
\hline Target $\mathbf{m} / \mathbf{z}:$ & 436.2097 & Result type: & Positive ions & Species: & {$[\mathrm{M}+\mathrm{Na}]^{+}$} \\
\hline \multicolumn{2}{|c|}{ Elements: } & \multicolumn{4}{|c|}{$\mathrm{C}(0-80) ; \mathrm{H}(0-120) ; \mathrm{O}(0-30) ; \mathrm{N}(0-5) ; \mathrm{Na}(0-5)$} \\
\hline \multicolumn{2}{|c|}{ Ion Formula } & \multicolumn{2}{|c|}{ Calculated $\mathbf{m} / \mathbf{z}$} & \multicolumn{2}{|c|}{ PPM Error } \\
\hline \multicolumn{2}{|c|}{$\mathrm{C} 24 \mathrm{H} 31 \mathrm{NNaO} 5$} & \multicolumn{2}{|c|}{436.2094} & \multicolumn{2}{|c|}{-0.59} \\
\hline
\end{tabular}




\section{Compound 16}

${ }^{1} \mathrm{H}$ NMR of compound $\mathbf{1 6}$ :

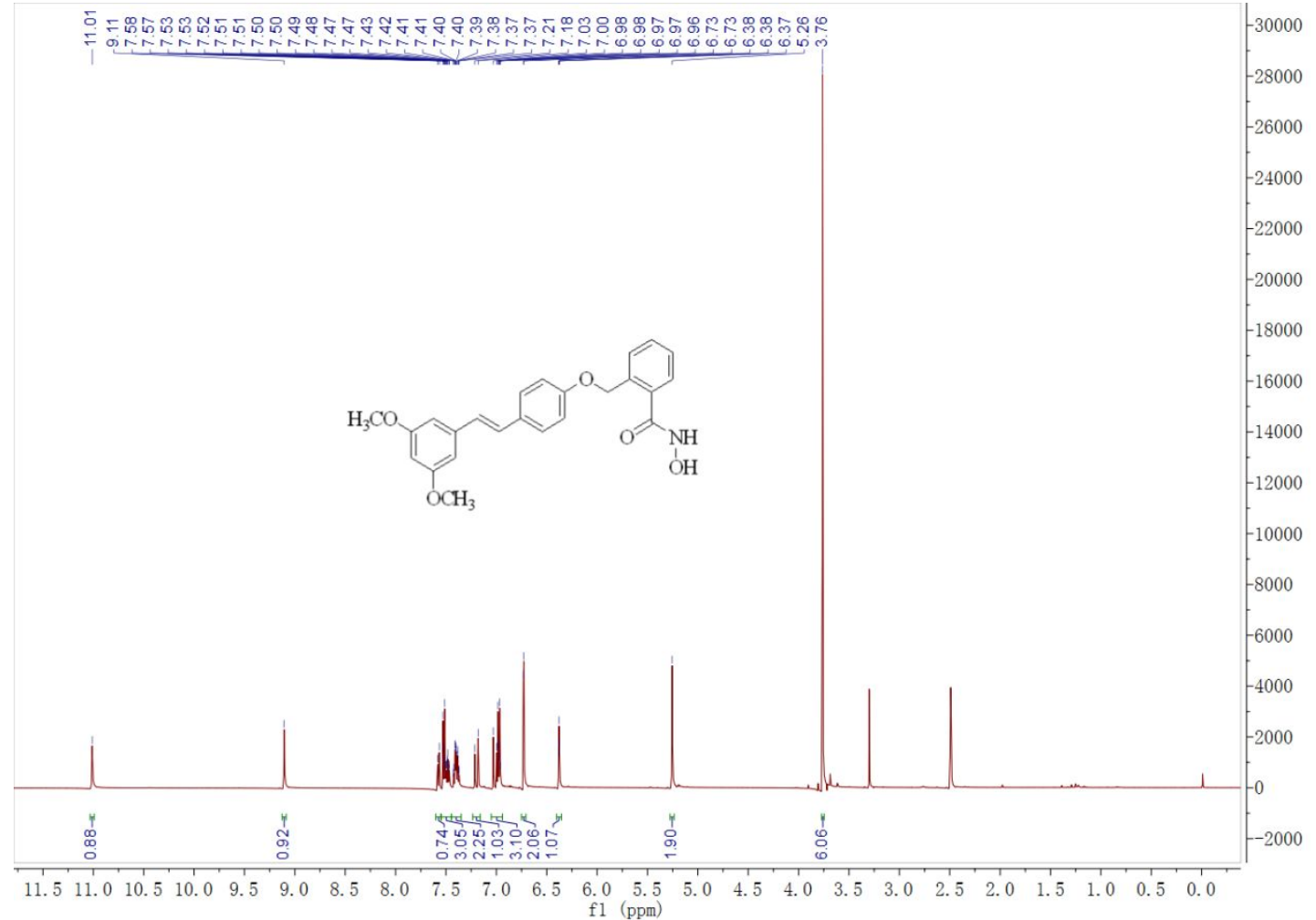


${ }^{13} \mathrm{C}$ NMR of compound $\mathbf{1 6}$ :

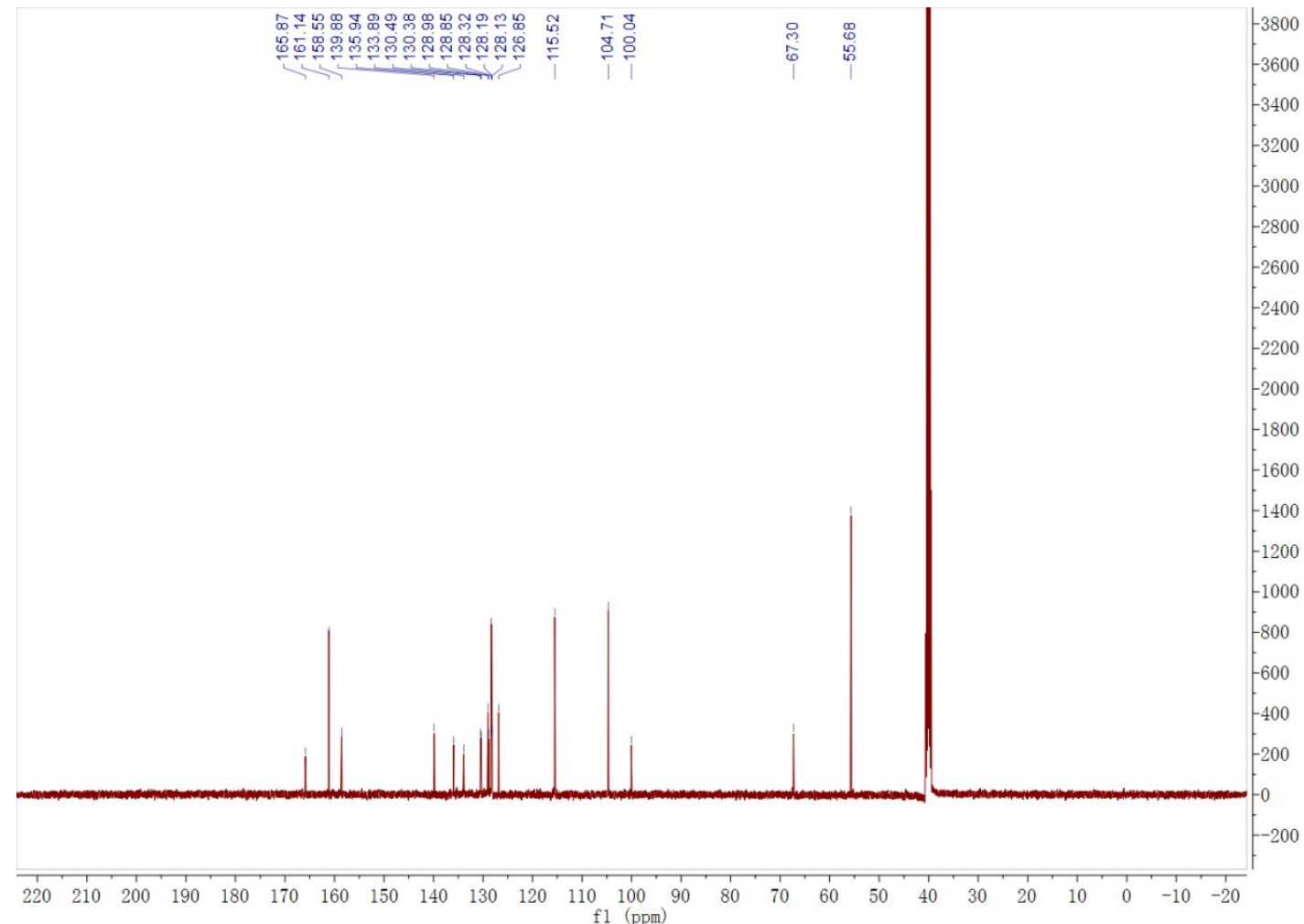

IR of compound 16

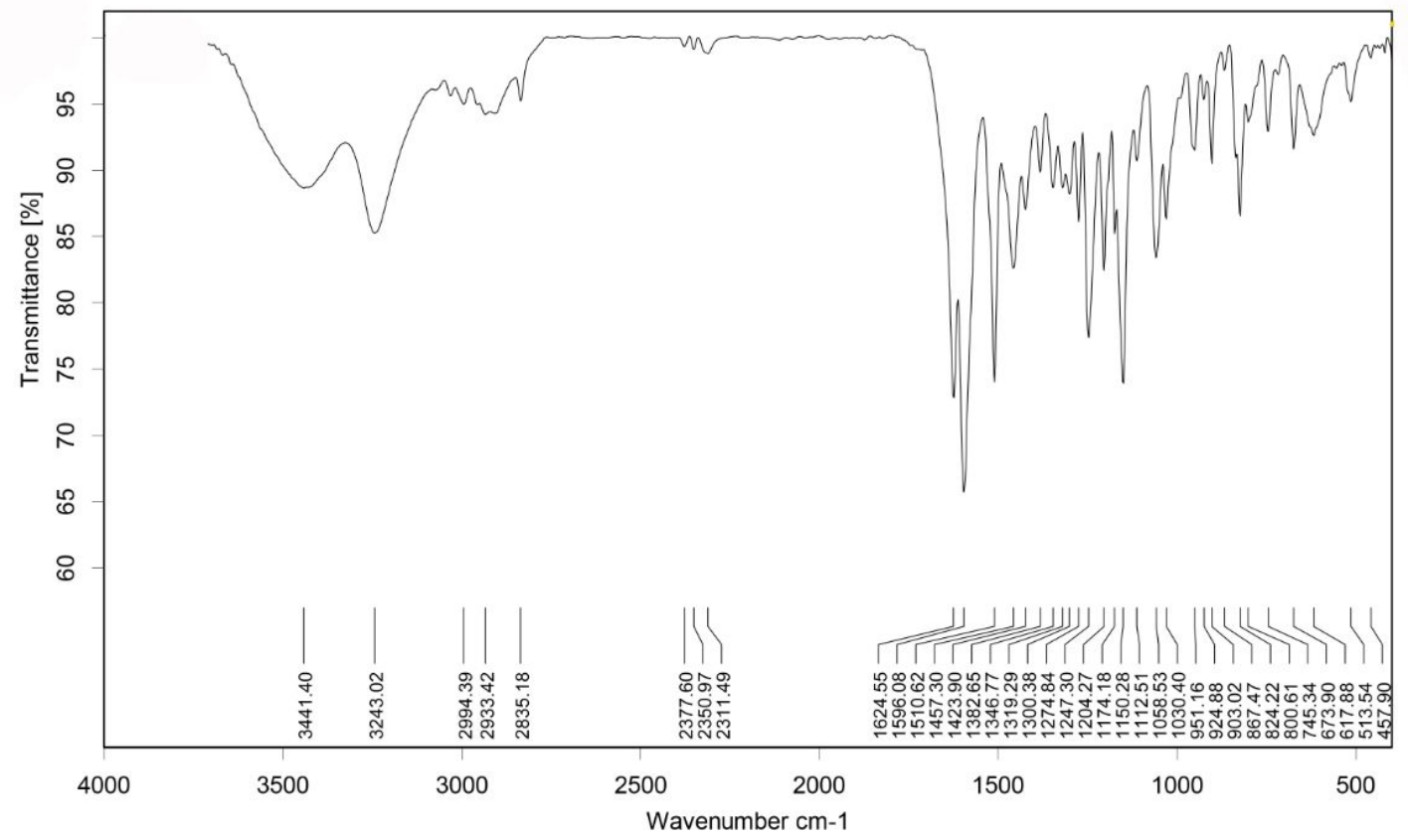


HRMS (ESI) of compound 16:

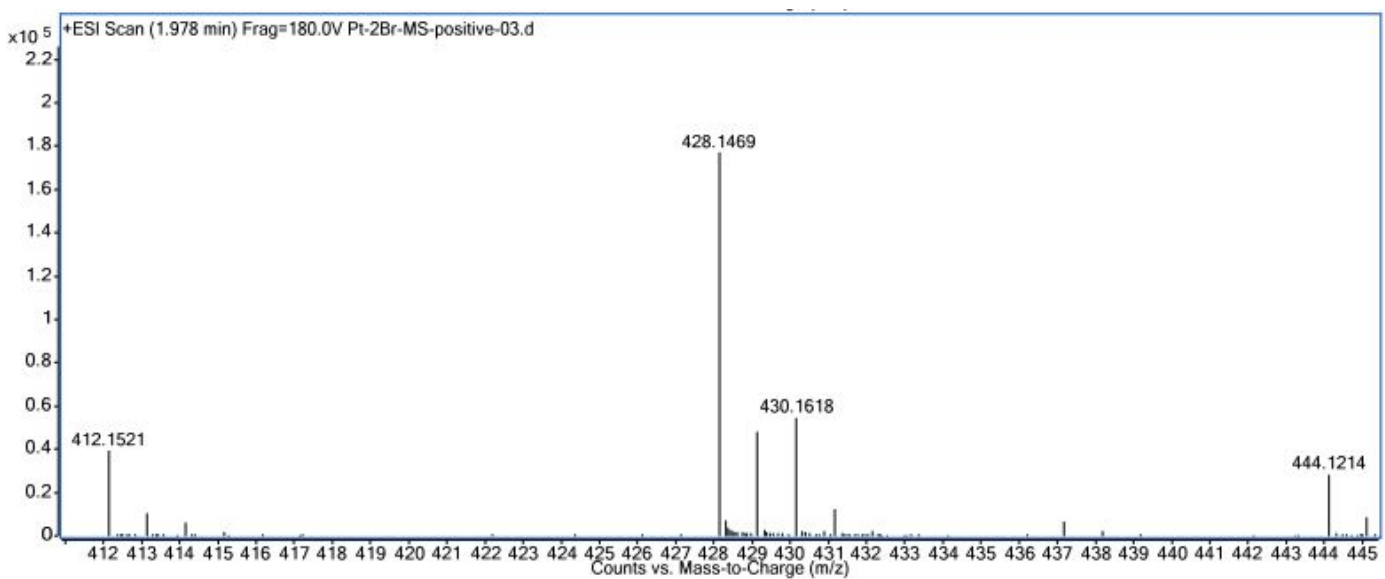

Elemental Composition Calculator

\begin{tabular}{|c|c|c|c|c|c|}
\hline Target $\mathbf{m} / \mathbf{z}$ : & 428.1469 & Result type: & Positive ions & Species: & {$[\mathrm{M}+\mathrm{Na}]^{+}$} \\
\hline \multicolumn{2}{|c|}{ Elements: } & \multicolumn{4}{|c|}{$\mathrm{C}(0-80) ; \mathrm{H}(0-120) ; \mathrm{O}(0-30) ; \mathrm{N}(0-5) ; \mathrm{Na}(0-5)$} \\
\hline \multicolumn{2}{|c|}{ Ion Formula } & \multicolumn{2}{|c|}{ Calculated $\mathbf{m} / \mathbf{z}$} & \multicolumn{2}{|c|}{ PPM Error } \\
\hline \multicolumn{2}{|c|}{$\mathrm{C} 24 \mathrm{H} 23 \mathrm{NNaO} 5$} & \multicolumn{2}{|c|}{428.1468} & \multicolumn{2}{|c|}{-0.14} \\
\hline
\end{tabular}


Compound 17

${ }^{1} \mathrm{H}$ NMR of compound $\mathbf{1 7}$ :

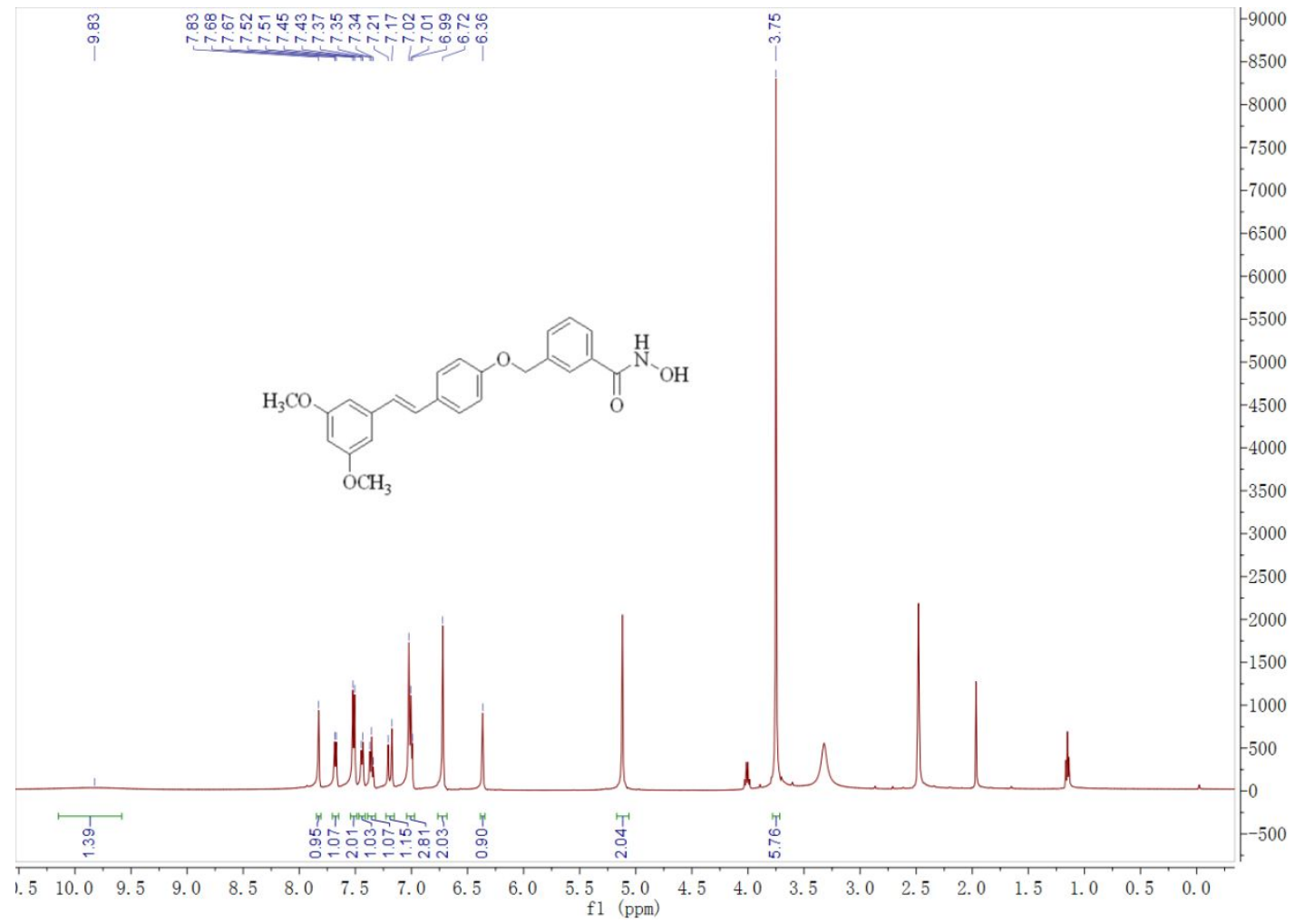


${ }^{13} \mathrm{C}$ NMR of compound $\mathbf{1 7}$ :

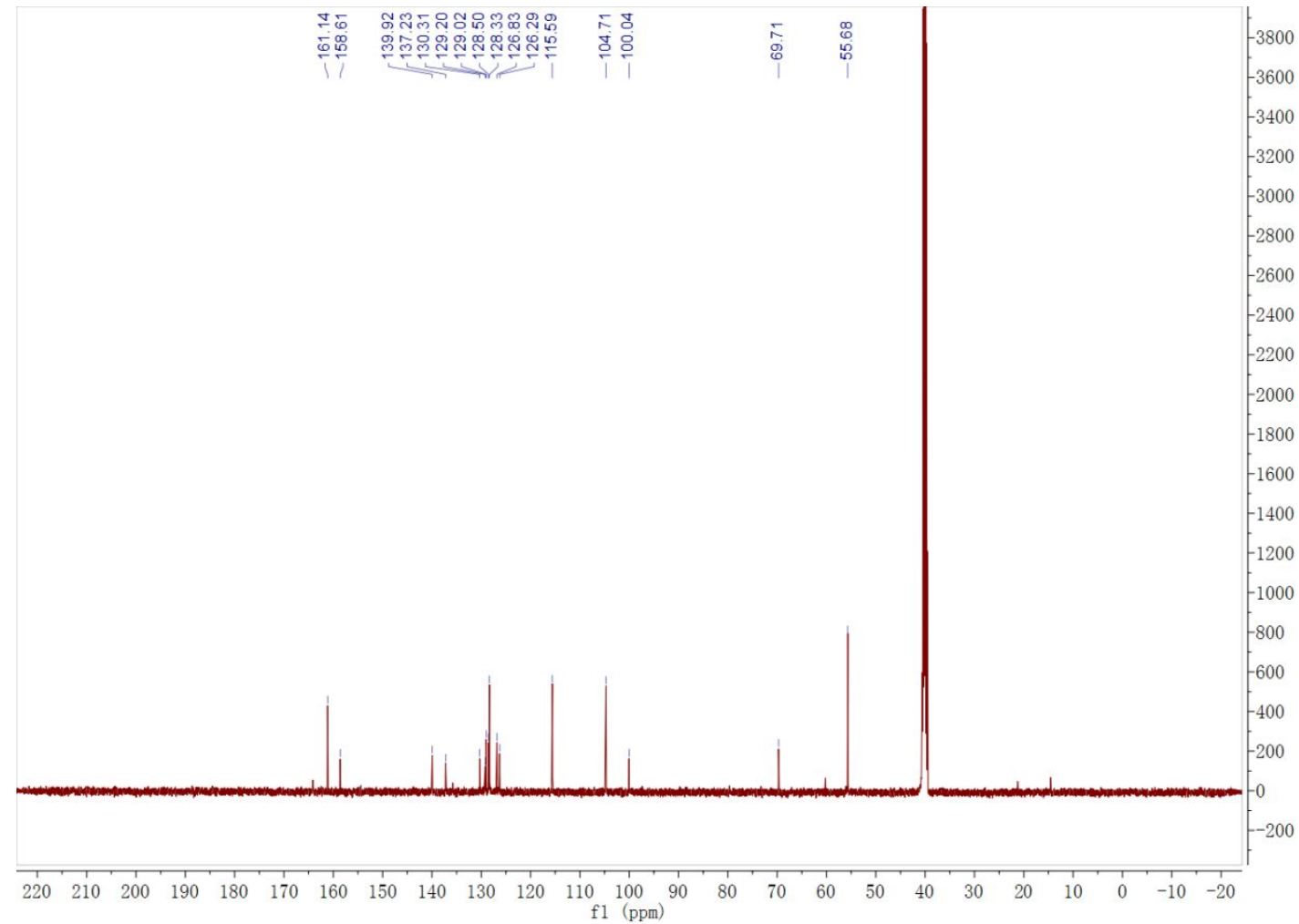

\section{IR of compound 17}

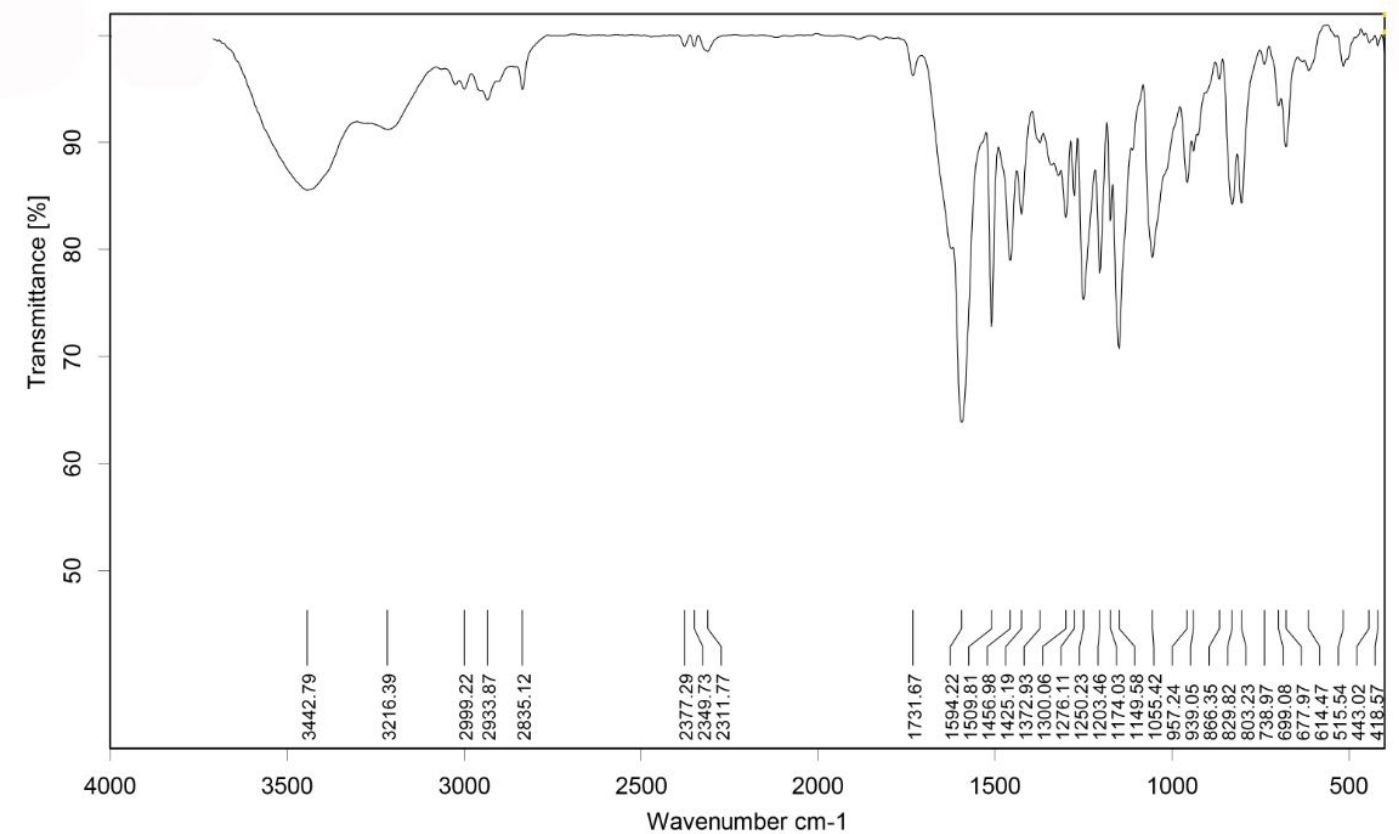


HRMS (ESI) of compound 17:

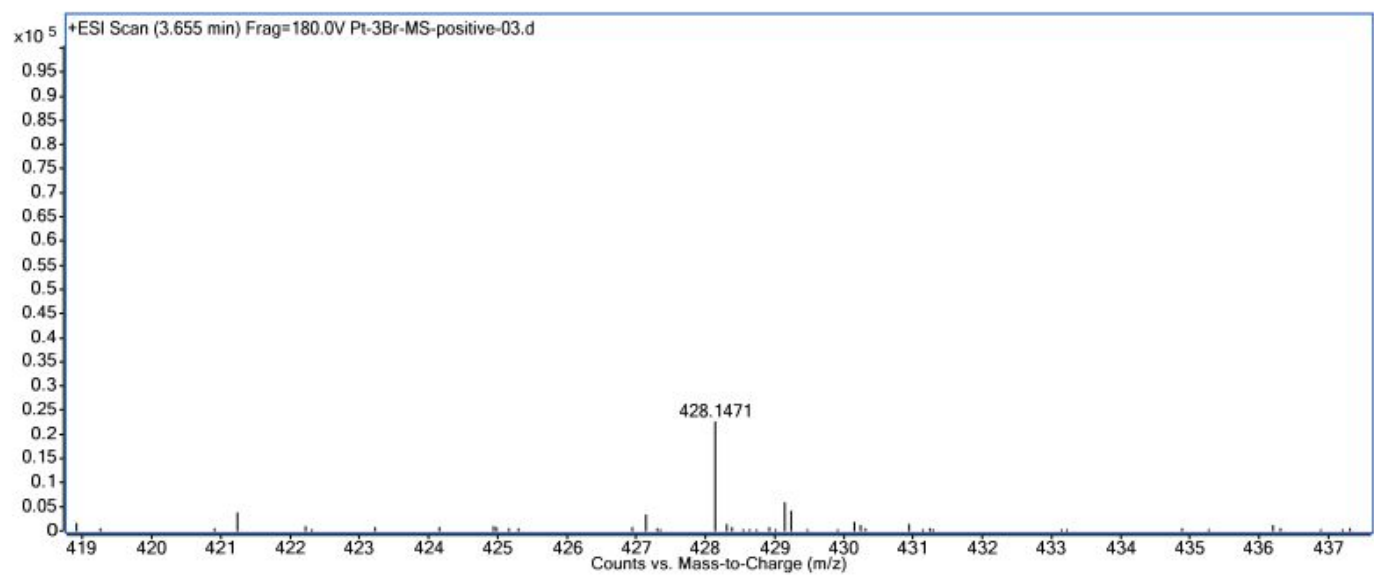

Elemental Composition Calculator

\begin{tabular}{|c|c|c|c|c|c|}
\hline Target $\mathbf{m} / \mathbf{z}$ : & 428.1471 & Result type: & Positive ions & Species: & {$[\mathrm{M}+\mathrm{Na}]^{+}$} \\
\hline \multicolumn{2}{|c|}{ Elements: } & \multicolumn{4}{|c|}{$\mathrm{C}(0-80) ; \mathrm{H}(0-120) ; \mathrm{O}(0-30) ; \mathrm{Na}(0-5) ; \mathrm{N}(0-5)$} \\
\hline \multicolumn{2}{|c|}{ Ion Formula } & \multicolumn{2}{|c|}{ Calculated $\mathrm{m} / \mathbf{z}$} & \multicolumn{2}{|c|}{ PPM Error } \\
\hline \multicolumn{2}{|c|}{$\mathrm{C} 24 \mathrm{H} 23 \mathrm{NNaO} 5$} & \multicolumn{2}{|c|}{428.1468} & \multicolumn{2}{|c|}{-0.52} \\
\hline
\end{tabular}


Compound 18

${ }^{1} \mathrm{H}$ NMR of compound $\mathbf{1 8}$ :

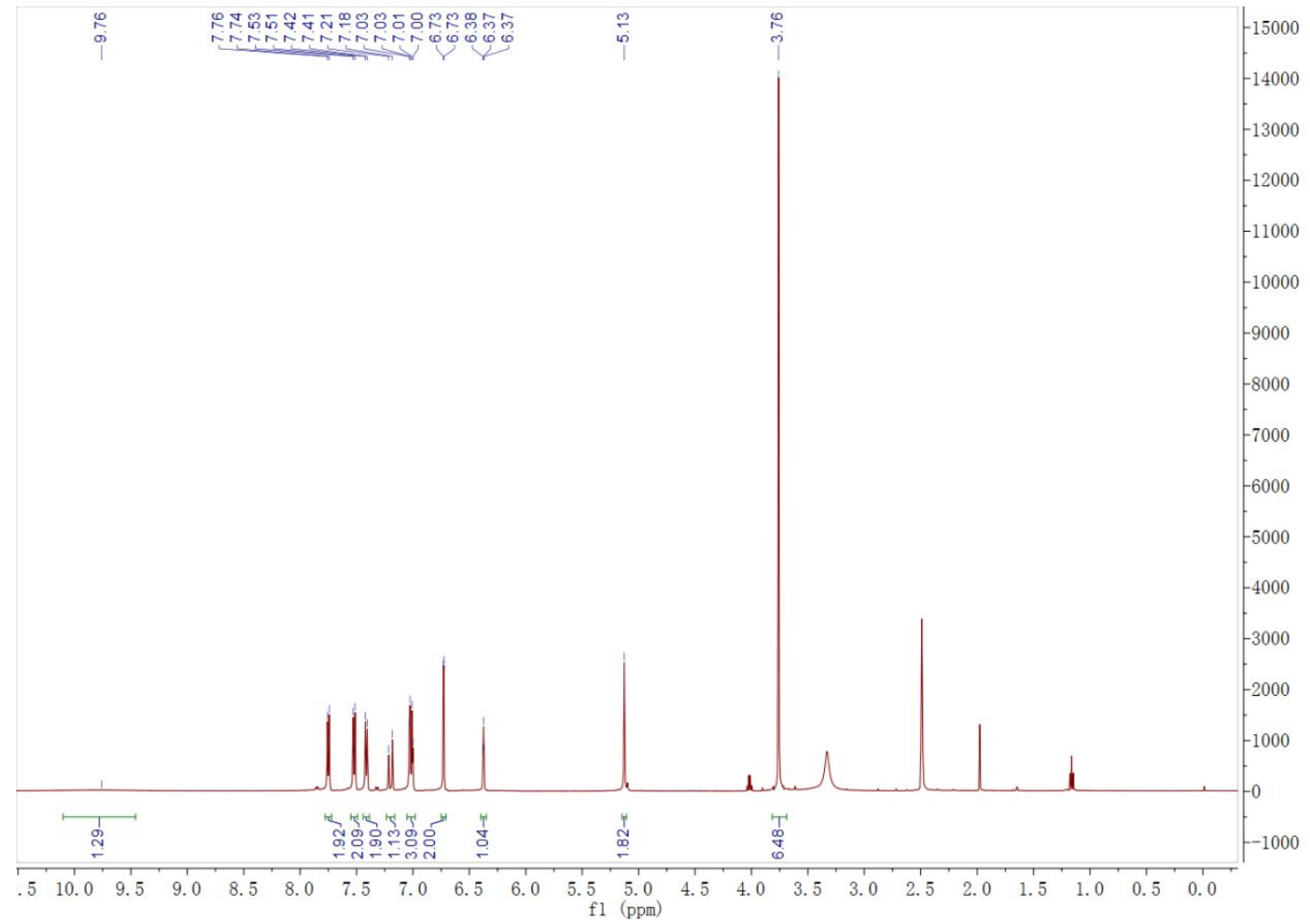


${ }^{13} \mathrm{C}$ NMR of compound $\mathbf{1 8}$ :

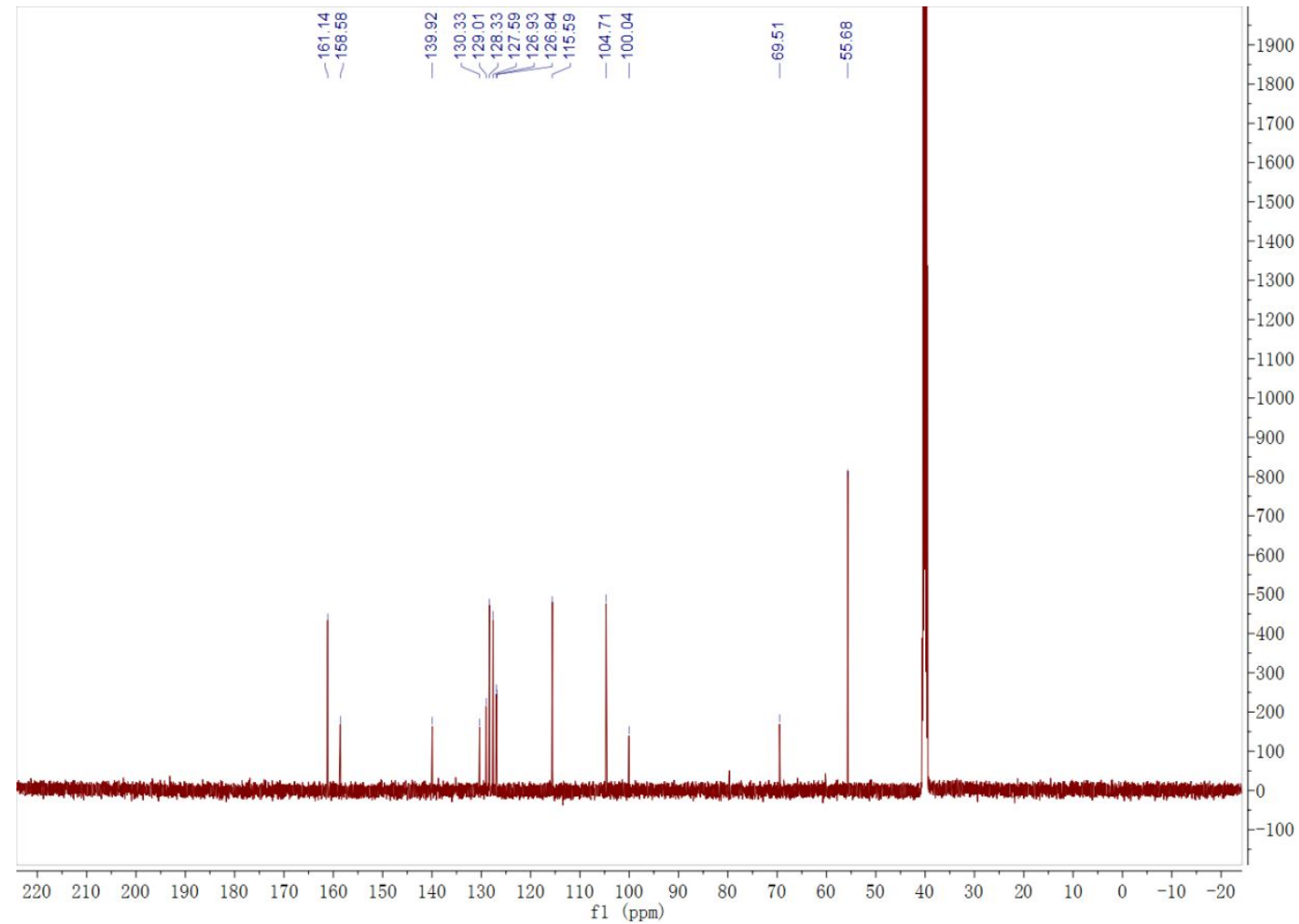

IR of compound $\mathbf{1 8}$

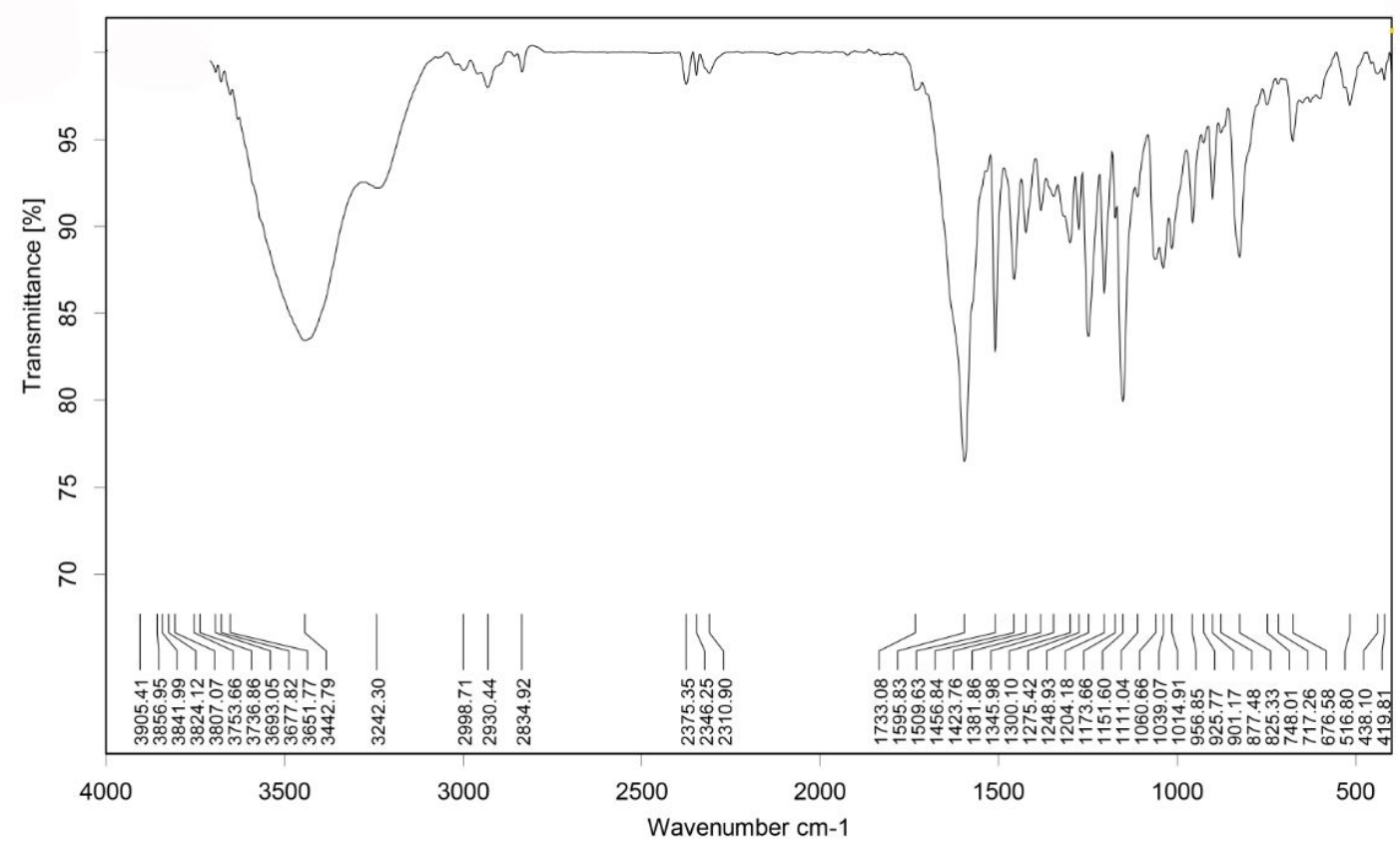


HRMS (ESI) of compound 18:

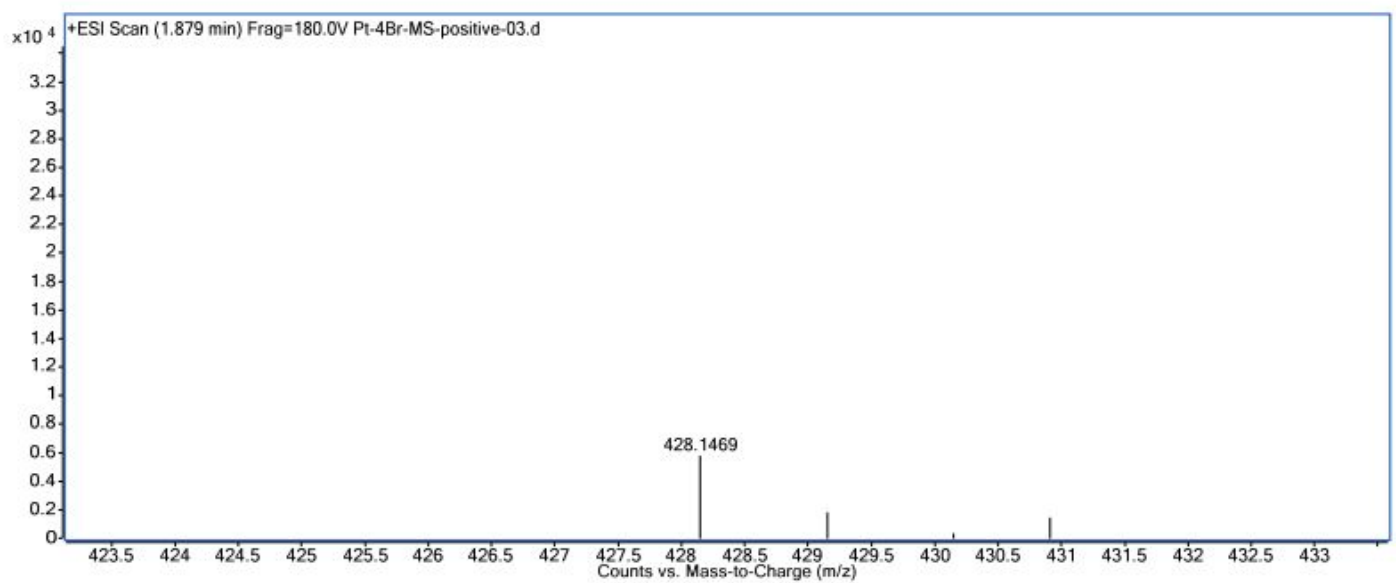

Elemental Composition Calculator

\begin{tabular}{|c|c|c|c|c|c|}
\hline Target $\mathbf{m} / \mathbf{z}$ : & 428.1469 & Result type: & Positive ions & Species: & {$[\mathrm{M}+\mathrm{Na}]^{+}$} \\
\hline \multicolumn{2}{|c|}{ Elements: } & \multicolumn{4}{|c|}{$\mathrm{C}(0-80) ; \mathrm{H}(0-120) ; \mathrm{O}(0-30) ; \mathrm{Na}(0-5) ; \mathrm{N}(0-5)$} \\
\hline \multicolumn{2}{|c|}{ Ion Formula } & \multicolumn{2}{|c|}{ Calculated $\mathbf{m} / \mathbf{z}$} & \multicolumn{2}{|c|}{ PPM Error } \\
\hline \multicolumn{2}{|c|}{$\mathrm{C} 24 \mathrm{H} 23 \mathrm{NNaO} 5$} & \multicolumn{2}{|c|}{428.1468} & \multicolumn{2}{|c|}{-0.11} \\
\hline
\end{tabular}


Compound 19

${ }^{1} \mathrm{H}$ NMR of compound 19:

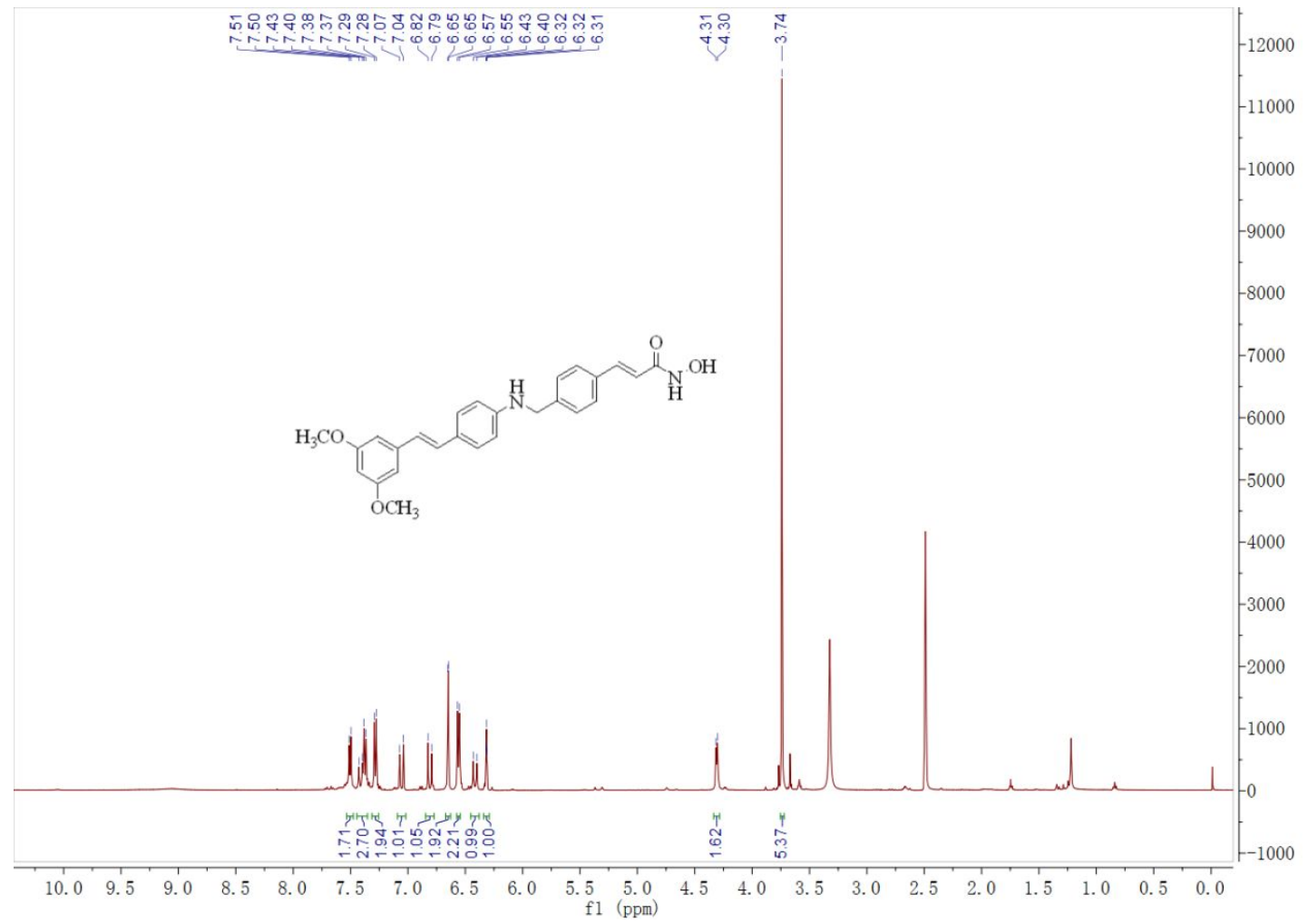


${ }^{13} \mathrm{C}$ NMR of compound $\mathbf{1 9}$ :

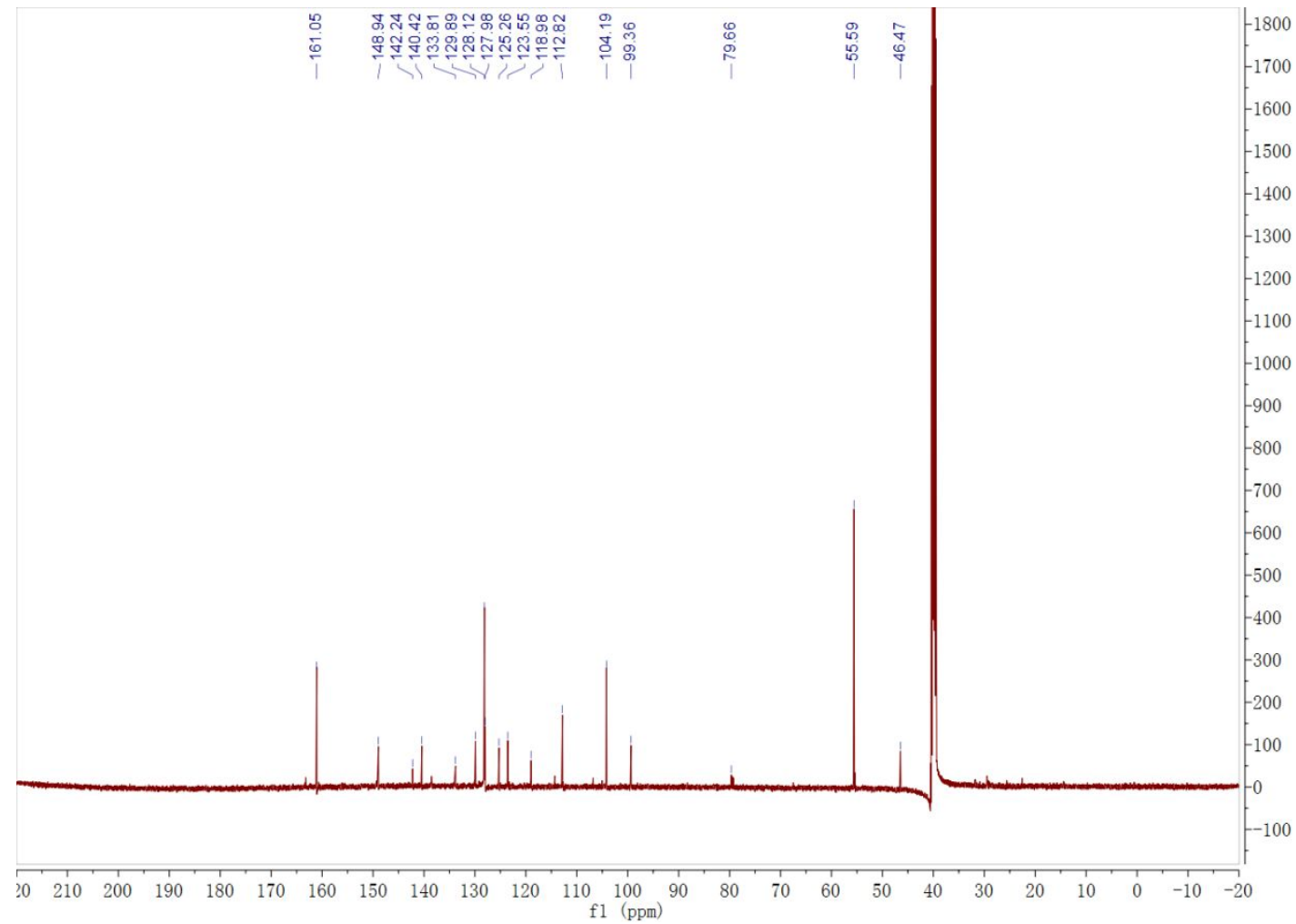

\section{IR of compound 19}

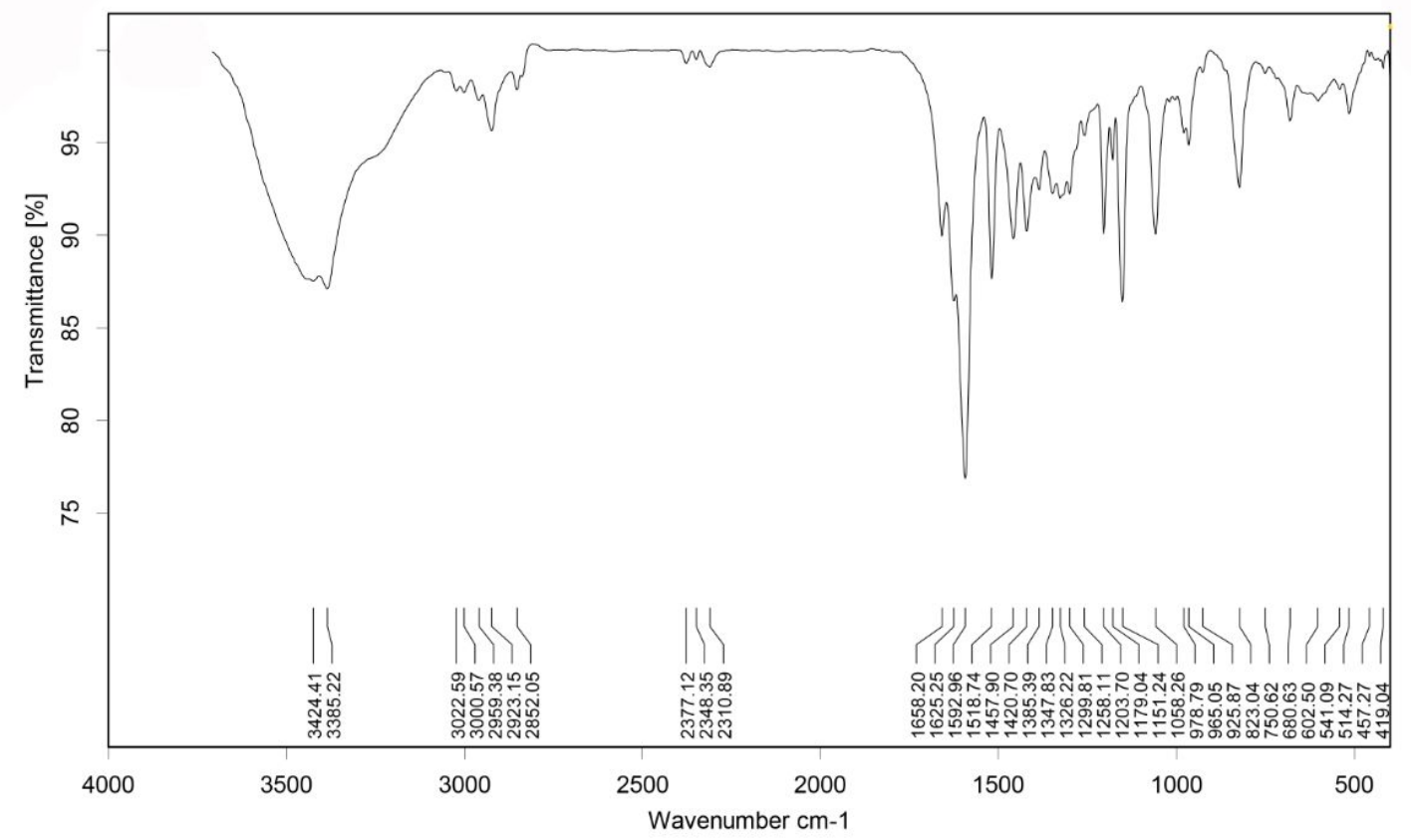


HRMS (ESI) of compound 19:

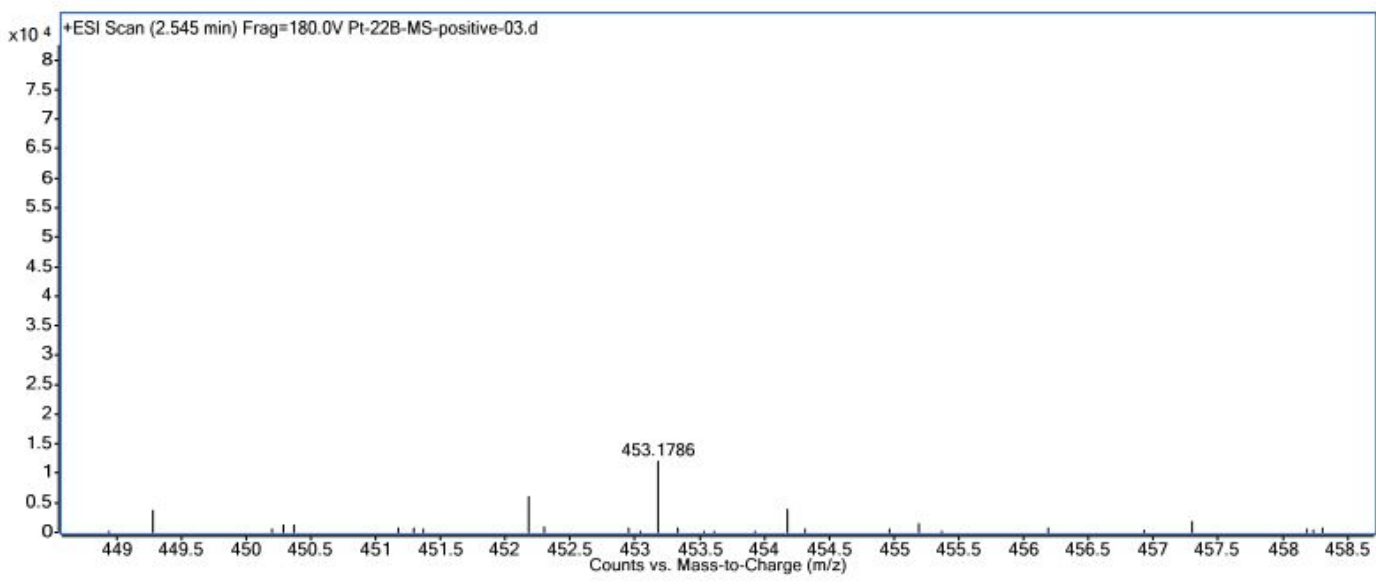

Elemental Composition Calculator

\begin{tabular}{|c|c|c|c|c|c|}
\hline Target $\mathbf{m} / \mathbf{z}$ : & 453.1786 & Result type: & Positive ions & Species: & {$[\mathrm{M}+\mathrm{Na}]^{+}$} \\
\hline \multicolumn{2}{|c|}{ Elements: } & \multicolumn{4}{|c|}{$\mathrm{C}(0-80) ; \mathrm{H}(0-120) ; \mathrm{O}(0-30) ; \mathrm{Na}(0-5) ; \mathrm{N}(0-5)$} \\
\hline \multicolumn{2}{|c|}{ Ion Formula } & \multicolumn{2}{|c|}{ Calculated $\mathrm{m} / \mathrm{z}$} & \multicolumn{2}{|c|}{ PPM Error } \\
\hline \multicolumn{2}{|c|}{$\mathrm{C} 26 \mathrm{H} 26 \mathrm{~N} 2 \mathrm{NaO} 4$} & \multicolumn{2}{|c|}{453.1785} & \multicolumn{2}{|c|}{-0.29} \\
\hline
\end{tabular}


A Mean Plasma Concentration-Time Curve
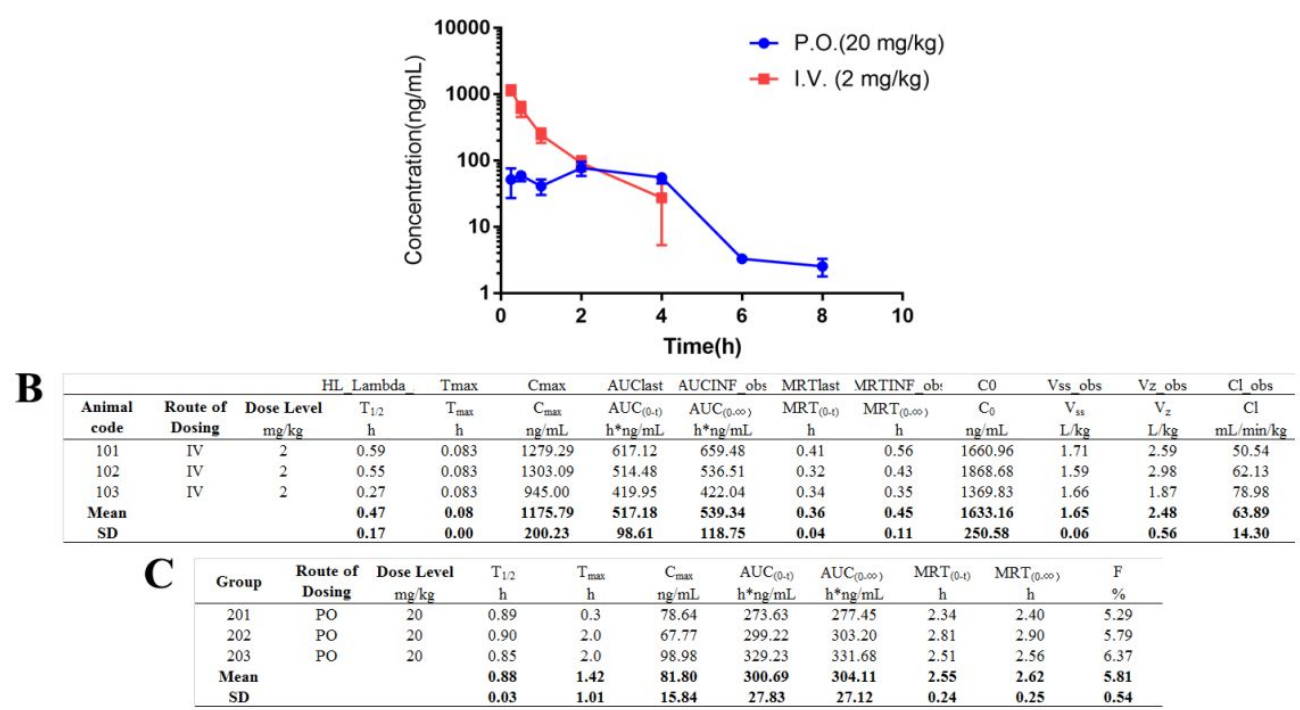

Figure S1. Pharmacokinetics of $\mathbf{1 4}$ in SD rats. (A) Semilogarithmic plot of mean plasma concentration versus time. (B) Intravenous PK parameters. (C) Oral PK parameters. 